\title{
Static and dynamic properties of dissipative particle dynamics
}

\author{
C. A. Marsh \\ Theoretical Physics, Oxford University, 1 Keble Road, Oxford OX1 3NP, United Kingdom
}

\author{
G. Backx and M. H. Ernst \\ Institute for Theoretical Physics, Utrecht University, Princetonplein 5, P.O. Box 80.006, 3508 TA Utrecht, The Netherlands
}

(Received 5 February 1997)

\begin{abstract}
The algorithm for the dissipative particle dynamics (DPD) fluid, the dynamics of which is conceptually a combination of molecular dynamics, Brownian dynamics, and lattice gas automata, is designed for simulating rheological properties of complex fluids on hydrodynamic time scales. This paper calculates the equilibrium and transport properties (viscosity, self-diffusion) of the thermostated DPD fluid explicitly in terms of the system parameters. It is demonstrated that temperature gradients cannot exist, and that there is therefore no heat conductivity. Starting from the $N$-particle Fokker-Planck, or Kramers equation, we prove an $H$ theorem for the free energy, obtain hydrodynamic equations, and derive a nonlinear kinetic equation (the FokkerPlanck-Boltzmann equation) for the single-particle distribution function. This kinetic equation is solved by the Chapman-Enskog method. The analytic results are compared with numerical simulations. [S1063-651X(97)00608-9]

PACS number(s): 47.11. $+\mathrm{j}, 51.10 .+\mathrm{y}, 83.70 . \mathrm{Hq}, 02.70 . \mathrm{Ns}$
\end{abstract}

\section{INTRODUCTION}

The interest of the last decade in dynamical and rheological properties of complex fluids has seen the introduction of several new numerical methods for carrying out computer simulations on hydrodynamic time scales, the simulation of which using molecular dynamics often results in intensive computational demands. These new techniques include (i) lattice gas cellular automata (LGCA) [1,2]; (ii) lattice Boltzmann equation (LBE) [3]; and (iii) dissipative particle dynamics (DPD).

The last method was introduced by Hoogerbrugge and Koelman [4], and was modified by Español and Warren [5] to ensure a proper thermal equilibrium state. The primary goal of this paper is a theoretical analysis and explicit calculation of transport and thermodynamic properties in terms of model parameters. This is highly relevant in view of the current interest in applications of DPD to systems such as flows past complex objects [4], concentrated colloidal suspensions [6,7], dilute polymer solutions [8,9], and phase separation [10].

The DPD algorithm models a fluid of $N$ interacting particles out of equilibrium and conserves mass and momentum. Position and velocity variables are continuous, as in molecular dynamics (MD), but time is updated in discrete steps $\delta t$, as in LGCA and LBE. The algorithm is a mixture of molecular dynamics, Brownian and Stokesian dynamics, and LGCA's, with a collision and a propagation step. In the collision step each particle interacts with all the particles inside an action sphere of radius $R_{0}$ through conservative forces $\mathbf{F}_{i j}$, dissipative forces $\mathbf{F}_{D, i j}$, which are proportional to both the step size $\delta t$ and a friction constant $\gamma$, and random forces $\mathbf{F}_{R, i j}$, which supply the energy lost by the damping. Here $i, j \in\{1,2, \ldots, N\}$ label the particles. In numerical simulations, this is implemented by simultaneously updating the velocities from their precollision value $\mathbf{v}_{i}$ to their postcollision value $\mathbf{v}_{i}^{*}$ according to the instantaneous forces ex- erted by all particles inside the action sphere. In the subsequent propagation step of fixed length $\delta t$ all particles move freely to their new positions $\mathbf{r}_{i}(t+\delta t)=\mathbf{r}_{i}(t)+\mathbf{v}_{i}^{*} \delta t$. There are no hard cores and the particles may be considered as completely interpenetrable. These softer interactions have the computational advantage $[4,8]$ of allowing particle motion on the order of a mean free path $l_{0}$ during each time step of fixed length $\delta t$. This represents a substantial advantage over event driven MD algorithms for hard sphere fluids, where the length $\delta t$ of the free propagation interval is on average much shorter, especially at fluid densities.

By ignoring some of the microscopic details of the interactions, which are presumably irrelevant for fluid dynamics, DPD has the advantages of LGCAs, but avoids the disadvantages of lacking Galilean invariance and of introducing spurious conservation laws. In fact, the "point particles" should not be considered as molecules in a fluid, but rather as clusters of particles that interact dissipatively [4,5]. The introduction of noise and dissipation represents a coarse-grained mesoscopic level of description and hydrodynamic behavior is expected at much smaller particle numbers than in conventional MD. If $t_{0} \simeq 1 / \gamma n R_{0}^{d}$ denotes the characteristic kinetic time scale in DPD, with $n=N / V$ the number density, $d$ the number of dimensions, and $\gamma$ the friction constant, then $t_{0}$ is considered to be large compared to any molecular time scale.

In this coarse-grained description the dominant interactions are the dissipative and random forces, whereas the conservative forces can be interpreted as weak forces of relatively long range and may be taken into account as a Vlasov mean field term in the kinetic equations. In addition, they can have the spurious effect of tending to force the DPD particles into "colloidal crystal" configurations, unless friction and noise are sufficiently large to prevent cooling into a lattice configuration $[4,5,11]$. In the second half of the paper, where we derive a kinetic equation for the single-particle distribution function, the conservative force will be neglected. This corresponds to the strong damping limit ( $\gamma$ large). The ran- 
dom forces act effectively as repulsive forces to prevent collapse of DPD particles.

A substantial contribution towards the understanding of the DPD fluid was given by Español and Warren [5], who derived a Fokker-Planck equation for the $N$-particle distribution function in the limit of continuous time $(\delta t \rightarrow 0)$. These authors also modified the original algorithm by imposing the detailed balance conditions, which guarantee the existence of the proper thermal Gibbs equilibrium, described by $\exp \left[-H / \theta_{0}\right]$ where $H$ is the Hamiltonian of the corresponding conservative system and $\theta_{0}=k_{B} T_{0}$ is the global equilibrium temperature. These results are briefly reviewed in Sec. II to establish the notation. Concerning the macroscopic evolution equations, Español formally established [12] the linearized Navier-Stokes equations and derived Green-Kubo formulas for the DPD transport coefficients using a MoriZwanzig projection operator technique. However, to date no quantitative evaluation of these formulas for DPD seems to exist. Hence, little is known explicitly about the approach to equilibrium, the validity of standard hydrodynamics (system size dependence, effects of generalized hydrodynamics), or about transport coefficients. For the transport coefficients, Hoogerbrugge and Koelman [4] have estimated the kinematic viscosity $\nu=\eta / \rho$, where $\eta$ is the shear viscosity and $\rho=n m$ is the mass density, as $\nu \sim \gamma n R_{0}^{d+2}$ with $n R_{0}^{d} \sim 1$. This result has recently been extended in $[13,11]$ to include the bulk viscosity by applying the "continuum approximation" to the discrete equations of motion for the DPD particles, following suggestions of Hoogerbrugge and Koelman [4]. In Sec. III we show how the free energy of the DPD fluid monotonically approaches its equilibrium value by proving an $H$ theorem for the Fokker-Planck equation of Español and Warren, and we make the connection with the detailed balance conditions derived in [5].

As a first step towards establishing the full nonlinear hydrodynamic equations we derive in Sec. IV the full macroscopic conservation laws for mass and momentum density, as well as the energy balance equation (details are given in the Appendix). The conceptual basis for the existence of hydrodynamic equations is the local equilibrium state, which in DPD is very different from that in a molecular fluid, because of the unusual role of the temperature. In Sec. V we study in a quantitative fashion the decay of the energy density $e(\mathbf{r}, t)$ and "kinetic" temperature $\theta(\mathbf{r}, t)$ towards thermal equilibrium with global temperature $\theta_{0}$, and we assess in what sense and on what time scale the DPD fluid describes an isothermal fluid out of equilibrium. This is done on the basis of a nonlinear kinetic equation-referred to as the Fokker-Planck-Boltzmann (FPB) equation-for the singleparticle distribution function $f(\mathbf{x}, t)$. It will be obtained from the first equation of the Bogoliubov-Born-Green-KirkwoodYvon (BBGKY) hierarchy for the DPD fluid in combination with the molecular chaos assumption.

By solving in Sec. VI the FPB equation in the hydrodynamic stage, using the Chapman-Enskog method, we derive the constitutive relations and the Navier-Stokes equation. This enables us to calculate in Sec. VII the transport coefficients of shear and bulk viscosity, as well as the selfdiffusion coefficient.

So far, we have not discussed the discrete time version of DPD, as implemented in actual simulations. They show a sensitive dependence of thermodynamic and transport properties on time step $\delta t[13,11,14]$. A promising step towards understanding the $\delta t$ dependence was recently taken by Marsh and Yeomans [14], who calculated the equilibrium temperature as a function of the step size, determined stability criteria for the step size, and validated their result by extensive numerical simulations. We shall not attempt to present here a systematic study of the different $O(\delta t)$ corrections to equilibrium distributions and transport properties, but postpone this to a later publication.

The paper ends in Sec. VIII with comments on the most important results and future prospects for DPD.

\section{THE FOKKER-PLANCK FORMALISM}

The dynamics of a DPD system defines the time evolution of an $N$-particle system, specified by a point $\Gamma$ $=\left\{\mathbf{x}_{i}=\left(\mathbf{v}_{i}, \mathbf{r}_{i}\right) \mid i=1,2, \ldots, N\right\}$ in phase space, in terms of stochastic differential equations. For a theoretical description it is more convenient to consider the equivalent FokkerPlanck equation, derived by Español and Warren [5].

To interpret the separate terms in the Fokker-Planck equation, it is instructive first to consider the analogous Kramers equation for the probability $P(\mathbf{v}, \mathbf{r}, t)$ of a single particle of mass $m$, having a phase description $\mathbf{x}=(\mathbf{v}, \mathbf{r})$ at time $t$ :

$$
\partial_{t} P+\mathbf{v} \cdot \frac{\partial}{\partial \mathbf{r}} P=-\frac{\mathbf{F}(\mathbf{r})}{m} \cdot \frac{\partial}{\partial \mathbf{v}} P+\gamma \frac{\partial}{\partial \mathbf{v}} \cdot \mathbf{v} P+\frac{\sigma^{2}}{2} \frac{\partial^{2}}{\partial \mathbf{v}^{2}} P .
$$

The three terms on the right can be interpreted as follows. The first term is an external conservative force $\mathbf{F}(\mathbf{r})=-\nabla V(r)$. The term involving the damping constant $\gamma$ corresponds to the Langevin force $-\gamma \mathbf{v}$ and the diffusive term with diffusion coefficient $\frac{1}{2} \sigma^{2}$ results from the random force $\sigma \hat{\xi}$ in the equivalent Langevin description, which reads

$$
\begin{gathered}
\frac{d \mathbf{r}}{d t}=\mathbf{v}, \\
\frac{d \mathbf{v}}{d t}=\frac{\mathbf{F}}{m}-\gamma \mathbf{v}+\sigma \hat{\xi},
\end{gathered}
$$

where $\sigma \hat{\xi}$ is Gaussian white noise with amplitude $\sigma$ and $\langle\hat{\xi}\rangle=0 \quad$ and $\quad\left\langle\hat{\xi}(t) \hat{\xi}\left(t^{\prime}\right)\right\rangle=I \delta\left(t-t^{\prime}\right)$, where $\quad I \quad$ is a $d$-dimensional unit tensor.

If we impose that the stationary solution of the Kramers equation be the Gibbs distribution: $P_{\text {eq }} \sim \exp \left\{-\left[\frac{1}{2} m \mathbf{v}^{2}\right.\right.$ $\left.+V(r)] / \theta_{0}\right\}$, then the diffusion coefficient must satisfy the following detailed balance (DB) condition:

$$
\sigma^{2}=\frac{2 \gamma \theta_{0}}{m}
$$

where $\theta_{0}=k_{B} T_{0}$ is the temperature in thermal equilibrium, measured in energy units.

The full Fokker-Planck equation derived by Español and Warren for the DPD system is a direct extension of the Kramers equation to $N$ interacting particles. The time evolution of the $N$-particle distribution function $P(\boldsymbol{\Gamma}, t)$ is governed by 


$$
\partial_{t} P=\left(\mathcal{L}_{C}+\mathcal{L}_{D}+\mathcal{L}_{R}\right) P,
$$

where the conservative, dissipative, and random parts of the evolution operator are defined, respectively, as

$$
\begin{aligned}
& \mathcal{L}_{C}=-\sum_{i}\left(\mathbf{v}_{i} \cdot \frac{\partial}{\partial \mathbf{r}_{i}}+\frac{\mathbf{F}_{i}}{m} \cdot \frac{\partial}{\partial \mathbf{v}_{i}}\right) \\
&=-\sum_{i} \mathbf{v}_{i} \cdot \frac{\partial}{\partial \mathbf{r}_{i}}-\frac{1}{2} \sum_{i, j \neq i} \frac{\mathbf{F}\left(\mathbf{R}_{i j}\right)}{m} \cdot\left(\frac{\partial}{\partial \mathbf{v}_{i}}-\frac{\partial}{\partial \mathbf{v}_{j}}\right), \\
& \mathcal{L}_{D}=\sum_{i, j \neq i} \gamma w_{D}\left(R_{i j}\right)\left(\hat{\mathbf{R}}_{i j} \cdot \frac{\partial}{\partial \mathbf{v}_{i}}\right)\left(\hat{\mathbf{R}}_{i j} \cdot \mathbf{v}_{i j}\right), \\
& \mathcal{L}_{R}=\sum_{i, j \neq i} \frac{\sigma^{2}}{2} w_{R}^{2}\left(R_{i j}\right)\left(\hat{\mathbf{R}}_{i j} \cdot \frac{\partial}{\partial \mathbf{v}_{i}}\right) \hat{\mathbf{R}}_{i j} \cdot\left(\frac{\partial}{\partial \mathbf{v}_{i}}-\frac{\partial}{\partial \mathbf{v}_{j}}\right) .
\end{aligned}
$$

The summations run over all particles and the only difference to the original [5] is that the parameters $\gamma$ and $\sigma$ have been scaled by the mass $m$ such that $\gamma$ has the dimensions of an inverse time. The three terms above are the $N$-particle extensions of the three terms on the right hand side of Eq. (1).

(1) The conservative part $\mathcal{L}_{C}$ results from the additive and central interparticle interactions due to a potential $V=\frac{1}{2} \sum_{i, j \neq i} \phi\left(R_{i j}\right)$ where $\mathbf{R}_{i j}=\mathbf{r}_{i}-\mathbf{r}_{j}$ is the relative position and a hat denotes a unit vector. It is the Liouville operator for the corresponding conservative system and in the limit of zero noise and friction, Eq. (5) reduces to the Liouville equation.

(2) The second term is analogous to the dissipative term in the Kramers equation. It accounts for the Langevin damping force between the pair $(i j)$, which is proportional to the friction constant $\gamma$ and to the component of the relative velocity $\mathbf{v}_{i j}$ along the line of centers $\hat{\mathbf{R}}_{i j}$, and is of finite range. This last property is described by a positive weighting function $w_{D}\left(R_{i j}\right)$ that is only nonvanishing inside an action sphere of finite radius $R_{0}$.

(3) The last term in Eq. (5) represents the random noise and should be compared with the diffusive term in Eq. (1). The random force $\sigma \hat{\xi}_{i j}$ between the pair $(i j)$ is directed along $\hat{\mathbf{R}}_{i j}$ and is proportional to $\sigma w_{R}\left(R_{i j}\right)$ where the weighting function $w_{R}\left(R_{i j}\right)$ is again only nonvanishing within a finite action sphere.

The ranges of the conservative, dissipative, and random forces may all be different as the model stands. Moreover, one of the essential properties of DPD is that its dynamics conserves total particle number $N$ and the total momentum $\mathbf{P}=\Sigma_{i} m \mathbf{v}_{i}$. Consequently, $\langle N\rangle$ $=\int d \mathbf{x} f(\mathbf{x}, t)$ and $\langle P\rangle=\int d \mathbf{x} m \mathbf{v} f(\mathbf{x}, t)$ are constants of the motion. The latter is always set equal to 0 as the total system is assumed to be at rest. Here $f(\mathbf{x}, t)$ is the single-particle distribution function.

In addition, we want to emphasize that microscopic momentum conservation is an essential property of a fluid model if it is to have a momentum density $\rho(\mathbf{r}, t) \mathbf{u}(\mathbf{r}, t)$ that is slowly varying in space and time. In contrast, we note that the energy of the system is not strictly conserved under the DPD algorithm. The equations for the mass, momentum, and

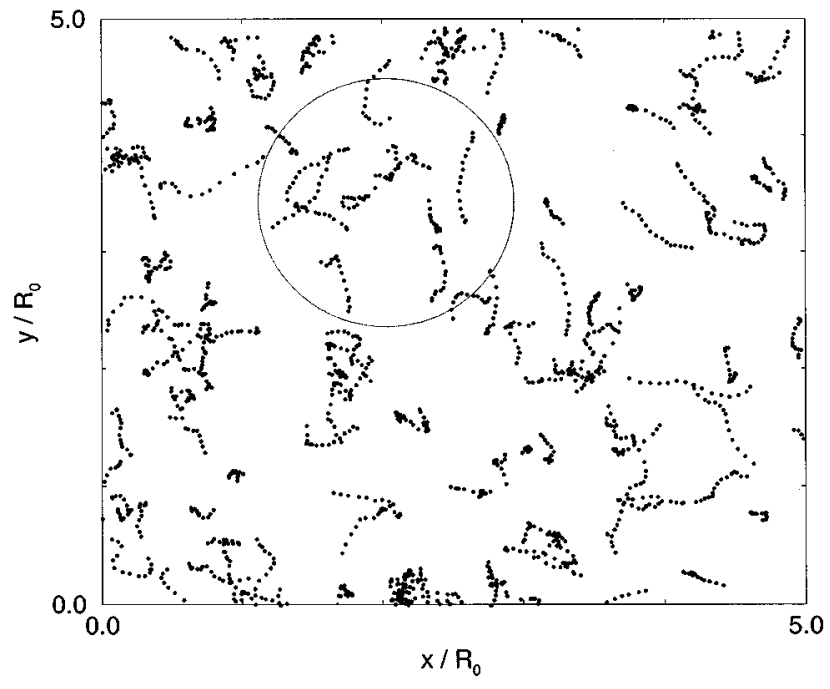

FIG. 1. Typical evolution of a particle configuration over a period of 20 time steps, showing that DPD interactions are "soft" as compared to hard core interparticle interactions. The circle with radius $R_{0}=4$ indicates the range of interaction.

energy density will be discussed more fully in Sec. IV. In typical applications the conservative force may be set equal to zero. The parameters $\gamma$ and $\sigma$ satisfy Eq. (3) and the weighting functions are chosen such that $w_{D}(r)=w_{R}^{2}(r)$, which constitute in combination with Eq. (3) the detailed balance conditions for the DPD system, as will be discussed in Sec. III. The density is typically chosen such that there are $5-10$ particles within an action sphere, which means that the instantaneous total force on any particle is small on average.

Figure 1 shows an enlargement of part of configuration space, showing a sequence of 20 consecutive particle positions evolving from a randomly chosen initial configuration. The trajectories are relatively smooth, in contrast to the discontinuous paths in hard core interactions, illustrating that the resultant force on each particle is relatively small at this parameter setting.

\section{AN $H$ THEOREM}

Consider the following functional of the $N$-particle distribution function $P(\boldsymbol{\Gamma}, t)$ :

$$
\mathcal{F} P]=\int d \boldsymbol{\Gamma} P(\boldsymbol{\Gamma}, t)\left\{H+\theta_{0} \ln P(\boldsymbol{\Gamma}, t)\right\},
$$

where $\theta_{0}=m \sigma^{2} / 2 \gamma$ and $H$ is the Hamiltonian of the corresponding conservative system:

$$
H=\sum_{i} \frac{1}{2} m \mathbf{v}_{i}^{2}+V=\sum_{i} \frac{1}{2} m \mathbf{v}_{i}^{2}+\frac{1}{2} \sum_{i, j \neq i} \phi\left(R_{i j}\right),
$$

$V$ is the potential energy and $\phi\left(R_{i j}\right)$ is the pair interaction. The functional can be interpreted as a sort of free energy $\mathcal{F}=E-\theta_{0} S$, where $E=\langle H\rangle$ is the average total energy and $S=-\langle\ln P\rangle$ yields the total entropy. The goal of this section is to show that $\mathcal{F}$ is a Lyapunov functional with $\partial_{t} \mathcal{F} \leqslant 0$ and to investigate the implications of this result for the equilibrium solution of the Fokker-Planck equation. 
The time derivative of Eq. (6) yields in combination with Eq. (4)

$$
\partial_{t} \mathcal{F}=\int d \boldsymbol{\Gamma}\left\{H+\theta_{0} \ln P+\theta_{0}\right\}\left(\mathcal{L}_{C}+\mathcal{L}_{D}+\mathcal{L}_{R}\right) P(\boldsymbol{\Gamma}, t) .
$$

We observe that the third term inside the curly brackets in Eq. (8) vanishes due to total probability conservation. Then consider the contribution $\left\{\partial_{t} \mathcal{F}\right\}_{C}$ to Eq. (8) due to the Liouville operator $\mathcal{L}_{C}$. Partial integration with respect to $\mathbf{r}_{i}$ and $\mathbf{v}_{i}$ yields directly

$$
\left\{\partial_{t} \mathcal{F}\right\}_{C}=-\int d \boldsymbol{\Gamma}\left\{P \mathcal{L}_{C} H+\theta_{0} \mathcal{L}_{C} P\right\}
$$

Here $\mathcal{L}_{C} H=\{H, H\}=0$ because the curly brackets represent Poisson brackets, as can easily be demonstrated. The second term in Eq. (9) reduces to surface terms in $\mathbf{r}_{i}$ and $\mathbf{v}_{i}$ and therefore vanishes too.

Next, we combine the remaining terms in Eq. (8) and perform a partial $\mathbf{v}_{i}$ integration, also symmetrizing the result over $i$ and $j$. The final result is

$$
\begin{aligned}
\partial_{t} \mathcal{F}= & -\frac{1}{2} m \gamma \int d \boldsymbol{\Gamma} \sum_{i, j \neq i}\left\{\hat{\mathbf{R}}_{i j} \cdot \mathbf{v}_{i j}+\frac{\theta_{0}}{m} \hat{\mathbf{R}}_{i j} \cdot\left(\frac{\partial}{\partial \mathbf{v}_{i}}-\frac{\partial}{\partial \mathbf{v}_{j}}\right) \ln P\right\} \\
& \times\left[w_{D}\left(R_{i j}\right) \hat{\mathbf{R}}_{i j} \cdot \mathbf{v}_{i j}+w_{R}^{2}\left(R_{i j}\right) \frac{\theta_{0}}{m} \hat{\mathbf{R}}_{i j} \cdot\left(\frac{\partial}{\partial \mathbf{v}_{i}}-\frac{\partial}{\partial \mathbf{v}_{j}}\right)\right] P .
\end{aligned}
$$

Now we make the following observation. If we choose

$$
w_{D}(r)=w_{R}^{2}(r) \equiv w(r),
$$

where $w(r)$ is an arbitrary positive function vanishing for $r>R_{0}$, then $\partial_{t} \mathcal{F} \leqslant 0$, as the right hand side of Eq. (10), can be cast into the form

$$
\partial_{t} \mathcal{F}=-\frac{1}{2} m \gamma \int d \boldsymbol{\Gamma} P \sum_{i, j \neq i} w\left(R_{i j}\right)\{\cdots\}^{2} \leqslant 0
$$

where $\{\cdots\}$ is the same as in Eq. (10). Note that the equality sign applies if and only if $P$ is the solution of Eq. (13) below. Consequently, the free-energy-type function $\mathcal{F} P]$ is a monotonically decreasing function of time, until it reaches equilibrium where $P=P_{\text {eq }}$, which is simply the solution of $\{\cdots\}=0$ for every pair $(i j)$ :

$$
\left\{\mathbf{v}_{i j}+\frac{\theta_{0}}{m}\left(\frac{\partial}{\partial \mathbf{v}_{i}}-\frac{\partial}{\partial \mathbf{v}_{j}}\right)\right\} P_{\mathrm{eq}}=0 .
$$

Changing variables to the relative velocities of the particles, it is easy to prove that the equilibrium distribution of the system is separable in the velocities, and has the general form

$$
P_{\mathrm{eq}}(\boldsymbol{\Gamma})=A\left(\mathbf{r}_{1}, \ldots, \mathbf{r}_{N}\right) \exp \left\{-\frac{1}{2 \theta_{0}} \sum_{i} m\left(\mathbf{v}_{i}-\mathbf{u}_{0}\right)^{2}\right\},
$$

where $\mathbf{u}_{0}$ is a constant independent of $\mathbf{r}_{i}$ and $t$. We will only consider macroscopic systems which are not in uniform motion at long times and consequently limit ourselves to $\mathbf{u}_{0}=\mathbf{0}$.

The function $P_{\text {eq }}(\boldsymbol{\Gamma})$ is also the stationary solution of the Fokker-Planck equation (4) if $A\left(\mathbf{r}_{1}, \ldots, \mathbf{r}_{N}\right)$ satisfies $\mathcal{L}_{C} A=0$. This yields the Gibbs distribution as the equilibrium solution:

$$
P_{\mathrm{eq}}(\boldsymbol{\Gamma})=\frac{1}{Z} \exp \left\{-\frac{H}{\theta_{0}}\right\},
$$

where $H$ is the Hamiltonian (7) of the system and $Z$ is a normalization constant. We assume that $P_{\text {eq }}$ is uniquely determined by the requirement that it satisfies Eqs. (13) and (4). Consequently the DPD system will always reach the same equilibrium state if left undriven, independent of the volume and number of particles. The temperature of this equilibrium state has a value $\theta_{0}=m \sigma^{2} / 2 \gamma$, which only depends on the parameters of the model. So DPD describes a system, thermostated at $\theta_{0}$ and with a free energy $\mathcal{F}\left[P_{\text {eq }}\right]$ at equilibrium. Note that, in contrast to the $H$ theorem for the Boltzmann equation (see, e.g., [15]), no molecular chaos approximation is required to derive this result for the DPD system.

In their original discussion [5], Español and Warren imposed that the Gibbs distribution be the stationary solution of the Fokker-Planck equation. The consequences of this requirement can be seen by inserting Eq. (15) into Eq. (4). It leads to the so-called detailed balance constraint

$$
w_{D}(r)=\frac{\sigma^{2} m}{2 \gamma \theta_{0}} w_{R}^{2}(r)=w_{R}^{2}(r)=w(r) .
$$

Consequently, the constraint imposed in Eq. (11) is the detailed balance constraint for DPD. The important result from the $H$ theorem is that it demonstrates that the Gibbs distribution (15) is the inevitable equilibrium distribution. Throughout the rest of the paper we shall restrict ourselves to dealing exclusively with DPD systems that obey the DB condition (16). If the DB condition is violated, no $H$ theorem can be derived and the Gibbs distribution is not a stationary solution of the FP equation for DPD. In this case, the stationary state of the system does not correspond to thermal equilibrium but to some driven state, which will in general exhibit long range spatial correlations (see, e.g., [16-18]).

The original version of DPD, introduced by Hoogerbrugge and Koelman [4], violates the DB requirement (16) and therefore its stationary distribution will not approach a Gibbs state but may exhibit spatial correlations, e.g., algebraic correlations $r^{-d}$ where $d$ is the number of dimensions, extending far beyond the ranges of the conservative, dissipative, and stochastic forces. This absence of thermal equilibrium is likely to be the reason for difficulties and inconsistencies discussed in $[4,5,12]$.

\section{MACROSCOPIC CONSERVATION AND BALANCE EQUATIONS}

In the preceding section we have established the existence of and approach to a thermal equilibrium state for the 
DPD system that obeys the detailed balance condition (16). In this section, we address the problem of how the quantities of macroscopic interest evolve in time towards the final equilibrium state, concentrating on the local mass density $\rho(\mathbf{r}, t)=m n(\mathbf{r}, t)$, the local momentum density $\rho(\mathbf{r}, t) \mathbf{u}(\mathbf{r}, t)$, and the local energy density $e(\mathbf{r}, t)$.

As discussed in Sec. II the microscopic dynamics of DPD conserve mass and momentum, and the corresponding macroscopic densities obey local conservation laws. As the total energy is not conserved under DPD, the evolution equation for the macroscopic energy density does not have the form of a local conservation equation, but contains source and sink terms corresponding to the random and dissipative forces, respectively. In the final equilibrium state, these will balance each other.

Consider a general macroscopic quantity $\langle A\rangle$, defined through

$$
\langle A\rangle=\int d \boldsymbol{\Gamma} A(\boldsymbol{\Gamma}) P(\boldsymbol{\Gamma}, t) .
$$

Its time evolution can be obtained from the Fokker-Planck equation (4) combined with the detailed balance condition (16), by multiplying the Fokker-Planck equation with $A(\boldsymbol{\Gamma}, t)$, integrating over all $\boldsymbol{\Gamma}$ space, and performing one or two partial integrations with respect to $\mathbf{v}_{i}$ and $\mathbf{v}_{j}$. The result is the general rate of change equation:

$$
\begin{aligned}
\partial_{t}\langle A\rangle= & \left\langle\sum_{i}\left(\mathbf{v}_{i} \cdot \frac{\partial}{\partial \mathbf{r}_{i}}+\frac{\mathbf{F}_{i}}{m} \cdot \frac{\partial}{\partial \mathbf{v}_{i}}\right) A\right\rangle \\
& -\gamma\left\langle\sum_{i, j \neq i} w\left(R_{i j}\right)\left\{\hat{\mathbf{R}}_{i j} \cdot \mathbf{v}_{i j}\right\}\left\{\hat{\mathbf{R}}_{i j} \cdot \frac{\partial}{\partial \mathbf{v}_{i}}\right\} A\right\rangle \\
& +\frac{\gamma \theta_{0}}{m}\left\langle\sum_{i, j \neq i} w\left(R_{i j}\right)\left\{\hat{\mathbf{R}}_{i j} \cdot \frac{\partial}{\partial \mathbf{v}_{i}}\right\}\right. \\
& \left.\times\left\{\hat{\mathbf{R}}_{i j} \cdot\left(\frac{\partial}{\partial \mathbf{v}_{i}}-\frac{\partial}{\partial \mathbf{v}_{j}}\right)\right\} A\right\rangle
\end{aligned}
$$

for any dynamic variable $A(\boldsymbol{\Gamma})$.

Consider the conserved mass density $\rho(\mathbf{r}, t)=m n(\mathbf{r}, t)$ and the momentum density $\rho(\mathbf{r}, t) \mathbf{u}(\mathbf{r}, t)$ defined through

$$
\begin{gathered}
n(\mathbf{r}, t)=\left\langle\sum_{i} \delta\left(\mathbf{r}-\mathbf{r}_{i}\right)\right\rangle=\int d \mathbf{v} f(\mathbf{v}, \mathbf{r}, t), \\
n \mathbf{u}(\mathbf{r}, t)=\left\langle\sum_{i} \mathbf{v}_{i} \delta\left(\mathbf{r}-\mathbf{r}_{i}\right)\right\rangle=\int d \mathbf{v} f(\mathbf{v}, \mathbf{r}, t) \mathbf{v} .
\end{gathered}
$$

It is convenient at this stage to introduce the single-particle and pair distribution functions, defined as

$$
\begin{gathered}
f(\mathbf{x}, t)=f(\mathbf{v}, \mathbf{r}, t)=\left\langle\sum_{i} \delta\left(\mathbf{x}-\mathbf{x}_{i}\right)\right\rangle, \\
f^{(2)}\left(\mathbf{x}, \mathbf{x}^{\prime}, t\right)=\left\langle\sum_{i, j \neq i} \delta\left(\mathbf{x}-\mathbf{x}_{i}\right) \delta\left(\mathbf{x}^{\prime}-\mathbf{x}_{j}\right)\right\rangle .
\end{gathered}
$$

Application of Eq. (18) to the conserved densities in Eq. (19) yields the macroscopic conservation laws:

$$
\begin{gathered}
\partial_{t} \rho=-\nabla \cdot \rho \mathbf{u}, \\
\partial_{t}(\rho \mathbf{u})=-\nabla \cdot(\rho \mathbf{u u}+\mathbf{\Pi}),
\end{gathered}
$$

where $\nabla=\partial / \partial \mathbf{r}$ and $\boldsymbol{\Pi}$ is the local pressure tensor or momentum flux density in the local rest frame of the fluid. The continuity equation has been derived by setting $A=\Sigma_{i} \delta\left(\mathbf{r}-\mathbf{r}_{i}\right)$ into Eq. (18). The only nonvanishing term is the one containing $\left(\partial / \partial \mathbf{r}_{i}\right) \delta\left(\mathbf{r}-\mathbf{r}_{i}\right)=-\nabla \delta\left(\mathbf{r}-\mathbf{r}_{i}\right)$, and the continuity equation follows at once. Derivation of the conservation equation for the momentum density proceeds along similar lines by choosing $A=\sum_{i} m \mathbf{v}_{i} \delta\left(\mathbf{r}-\mathbf{r}_{i}\right)$. Details of the latter derivation are given in the Appendix where it is shown that

$$
\boldsymbol{\Pi}(\mathbf{r}, t)=\Pi_{K}(\mathbf{r}, t)+\Pi_{C}(\mathbf{r}, t)+\Pi_{D}(\mathbf{r}, t),
$$

with kinetic $(K)$, collisional transfer $(C)$, and dissipative $(D)$ contributions:

$$
\begin{gathered}
\Pi_{K}=\int d \mathbf{v} m \mathbf{V V} f(\mathbf{v}, \mathbf{r}, t), \\
\Pi_{C}=\frac{1}{2} \int d \mathbf{v} d \mathbf{v}^{\prime} \int d \mathbf{R R F}(\mathbf{R}) \bar{f}^{(2)}\left(\mathbf{v}, \mathbf{r}, \mathbf{v}^{\prime}, \mathbf{r}^{\prime}, t\right), \\
\boldsymbol{\Pi}_{D}=-\frac{1}{2} m \gamma \int d \mathbf{v} d \mathbf{v}^{\prime} \int d \mathbf{R} w(R)\left\{\mathbf{R} \cdot\left(\mathbf{v}-\mathbf{v}^{\prime}\right)\right\} \hat{\mathbf{R}} \hat{\mathbf{R}} \\
\times \bar{f}^{(2)}\left(\mathbf{v}, \mathbf{r}, \mathbf{v}^{\prime}, \mathbf{r}^{\prime}, t\right),
\end{gathered}
$$

with $\mathbf{R}=\mathbf{r}-\mathbf{r}^{\prime}$. The kinetic flux contains the so-called peculiar velocity $\mathbf{V}=\mathbf{v}-\mathbf{u}(\mathbf{r}, t)$, and $\bar{f}^{(2)}$ is the spatially averaged pair distribution function:

$$
\bar{f}^{(2)}\left(\mathbf{v}, \mathbf{r}, \mathbf{v}^{\prime}, \mathbf{r}, t\right)=\int_{0}^{1} d \lambda f^{(2)}\left(\mathbf{v}, \mathbf{r}+\lambda \mathbf{R}, \mathbf{v}^{\prime}, \mathbf{r}+(\lambda-1) \mathbf{R}, t\right) .
$$

The kinetic and collisional transfer contributions to the momentum flux in Eqs. (22) and (23) are present in any particle model with conservative forces. They are dominant in systems with sufficiently high density-dense gases and liquids-where the potential energy contributions are nonnegligible with respect to the kinetic fluxes.

The explicit form for these collisional transfer contributions is given in the literature for several cases: smooth potentials [19], elastic hard spheres [20], or inelastic hard spheres [21]. The dissipative contribution $\boldsymbol{\Pi}_{D}$ results from the Langevin-type damping forces between the particles. The random forces do not contribute to the momentum flux.

The $H$ theorem, derived in Sec. III, guarantees the approach to thermal equilibrium, where the distribution functions take the form

$$
\begin{gathered}
f(\mathbf{x})=n_{0} \varphi_{0}(\mathbf{v})=n_{0}\left(\frac{m}{2 \pi \theta_{0}}\right)^{d / 2} \exp \left\{-\frac{m \mathbf{v}^{2}}{2 \theta_{0}}\right\}, \\
f^{(2)}\left(\mathbf{x}, \mathbf{x}^{\prime}\right)=n_{0}^{2} \varphi_{0}(\mathbf{v}) \varphi_{0}\left(\mathbf{v}^{\prime}\right) g\left(\left|\mathbf{r}-\mathbf{r}^{\prime}\right|\right),
\end{gathered}
$$


where $g(R)$ is the pair distribution function in thermal equilibrium, $n_{0}=N / V$ is the number density, and $\varphi_{0}(\mathbf{v})$ the Maxwellian velocity distribution.

The sum of the kinetic and collisional transfer contributions reduces to the equilibrium pressure and the dissipative contribution vanishes. Thus $\boldsymbol{\Pi}=p_{0} I$ with $p_{0}$ given by the virial theorem:

$$
p_{0}=n_{0} \theta_{0}-\frac{n_{0}^{2}}{2 d} \int d \mathbf{R} R \frac{d \phi(R)}{d R} g(R),
$$

where

$$
\mathbf{F}(\mathbf{R})=-\hat{\mathbf{R}} \frac{d \phi(R)}{d R} .
$$

Away from global equilibrium, the pressure tensor $\boldsymbol{\Pi}(\mathbf{r}, t)$ will contain the local equilibrium pressure and terms involving the viscosities. However, before the Navier-Stokes equations, or more generally, the full set of hydrodynamic equations, can be derived the concept of local equilibriumwhich forms the conceptual basis of slow hydrodynamic evolution - has to be reexamined, as the energy is no longer a conserved quantity. This can only be done after identifying the slow and fast relaxation modes in DPD, on the basis of a kinetic equation. This will be done in Sec. V.

Next we consider the energy density, defined as

$$
\begin{aligned}
e(\mathbf{r}, t)= & \left\langle\sum_{i} \epsilon_{i}(\mathbf{v}) \delta\left(\mathbf{r}-\mathbf{r}_{i}\right)\right\rangle \\
= & \int d \mathbf{v} \frac{1}{2} m \mathbf{v}^{2} f(\mathbf{x}, t)+\frac{1}{2} \int d \mathbf{v} d \mathbf{v}^{\prime} \\
& \times \int d \mathbf{R} \phi(R) f^{(2)}\left(\mathbf{v}, \mathbf{r}, \mathbf{v}^{\prime}, \mathbf{r}-\mathbf{R}, t\right),
\end{aligned}
$$

where $\epsilon_{i}(\mathbf{v})=\frac{1}{2} m V_{i}^{2}+\sum_{i \neq j} \phi\left(R_{i j}\right)$ is the microscopic energy per particle. Use of the rate of change equation (18) leads after some lengthy algebra to the energy balance equation, as discussed in the Appendix. It reads

$$
\partial_{t} e=-\nabla \cdot \mathbf{q}+\Gamma
$$

Here the explicit form of the source term [22] is

$$
\begin{aligned}
\Gamma(\mathbf{r}, t)= & \gamma\left(\sum_{i, j \neq i} w\left(R_{i j}\right)\left\{\theta_{0}-\frac{m}{2}\left\{\hat{\mathbf{R}}_{i j} \cdot\left(\mathbf{v}_{i}-\mathbf{v}_{j}\right)\right\}^{2}\right\}\right. \\
& \left.\times \delta\left(\mathbf{r}-\mathbf{r}_{i}\right)\right\rangle
\end{aligned}
$$

where the term proportional to $\theta_{0}$ is a source resulting from the random force, and the term with the minus sign is a sink resulting from the Langevin-type damping forces. In global equilibrium, the source and sink terms balance one another and $\Gamma_{\mathrm{eq}}=0$. The heat current $\mathbf{q}$, given explicitly in Eqs. (A12)-(A15) of the Appendix contains the standard kinetic and collisional transfer contributions due to conservative forces, as well as dissipative contributions analogous to $\boldsymbol{\Pi}_{D}$ in Eq. (23), and $\mathbf{q}$ vanishes in global equilibrium.

If $\Gamma$ were set equal to zero, Eq. (29) would have the generic form of the energy balance equation in ordinary hydrodynamics, where the heat current would contain a term proportional to the temperature gradient. As will become apparent in later sections, this is not the case in the DPD system. Although Eq. (29) looks like a macroscopic equation for the energy balance in the presence of sources and sinks, it loses its physical significance after a relaxation time $t_{0}$, in which $e(\mathbf{r}, t) \rightarrow(d / 2) \theta_{0} n(\mathbf{r}, t)$, and Eq. (29) reduces to the continuity equation. This will be discussed in Sec. VI, below Eq. (41).

One may also derive a balance equation for the free energy density, which would be a local version of the $H$ theorem of Sec. III or of the corresponding one of Sec. V for the Fokker-Planck-Boltzmann equation. It would enable one to identify the irreversible entropy production. A similar balance equation for the entropy density in a dilute gas can be derived from the Boltzmann equation [23].

\section{FOKKER-PLANCK-BOLTZMANN EQUATION}

In this section we derive an approximate kinetic equation, referred to as the Fokker-Planck-Boltzmann equation, for the single-particle distribution function $f(\mathbf{x}, t)$, which is based on the molecular chaos assumption and has a collision term which is quadratic in $f(\mathbf{x}, t)$.

Moreover, from here on the conservative forces will be neglected, which corresponds to the strong damping limit ( $\gamma$ large). Numerical measurements of the two-particle correlation function [11] have demonstrated that the inclusion of the conservative force can result in the formation of crystalline ordering, which is an undesirable feature in the current context of fluid dynamics or rheology of complex fluids. However, these effects may prove important to the ongoing investigation into the static properties of DPD.

This section is organized as follows. We start by deriving the first equation of the BBGKY hierarchy, which relates $\partial_{t} f$ to the pair function $f^{(2)}\left(\mathbf{x}, \mathbf{x}^{\prime}, t\right)$. Then the molecular chaos assumption

$$
f^{(2)}\left(\mathbf{x}, \mathbf{x}^{\prime}, t\right) \simeq f(\mathbf{x}, t) f\left(\mathbf{x}^{\prime}, t\right)
$$

yields a closed equation, the FPB equation, which again satisfies an $H$ theorem. Next we analyze the local equilibrium solution of the kinetic equation, which provides the conceptual basis for the existence of hydrodynamic equations and transport coefficients, as well as the justification for solving this kinetic equation for finding the "normal solution" by means of the Chapman-Enskog method.

The first equation of the BBGKY hierarchy can be derived directly by applying Eq. (18) to the $\mu$-space density:

$$
\hat{f}(\mathbf{x})=\sum_{i} \delta\left(\mathbf{x}-\mathbf{x}_{i}\right)
$$

Its average yields $f(\mathbf{x}, t)$ on account of Eq. (20). The resulting equation of motion is 


$$
\begin{aligned}
\partial_{t} f= & \int d \boldsymbol{\Gamma} P(\boldsymbol{\Gamma}, t)\left\{\sum_{i} \frac{\partial \hat{f}}{\partial \mathbf{r}_{i}} \cdot \mathbf{v}_{i}+\sum_{i, j \neq i} \gamma w\left(R_{i j}\right)\right. \\
& \left.\times\left[-\mathbf{v}_{i j} \frac{\partial}{\partial \mathbf{v}_{i}}+\frac{\theta_{0}}{m} \frac{\partial}{\partial \mathbf{v}_{i}}\left(\frac{\partial}{\partial \mathbf{v}_{i}}-\frac{\partial}{\partial \mathbf{v}_{j}}\right)\right] \hat{f}: \hat{\mathbf{R}}_{i j} \hat{\mathbf{R}}_{i j}\right\},
\end{aligned}
$$

where the (:) contraction of tensors is defined by $\mathrm{A}: \mathrm{B}=\Sigma_{\alpha \beta} A_{\alpha \beta} B_{\beta \alpha}$, with $\alpha, \beta$ denoting Cartesian components of vectors or tensors. The equation can be further simplified to

$$
\begin{aligned}
\partial_{t} f= & -\nabla \cdot\left\langle\sum_{i} \mathbf{v}_{i} \delta\left(\mathbf{x}-\mathbf{x}_{i}\right)\right\rangle \\
& +\gamma \frac{\partial}{\partial \mathbf{v}} \cdot\left\langle\sum_{i, j \neq i} \delta\left(\mathbf{x}-\mathbf{x}_{i}\right) w\left(R_{i j}\right) \hat{\mathbf{R}}_{i j}\left\{\hat{\mathbf{R}}_{i j} \cdot \mathbf{v}_{i j}\right\}\right\rangle \\
& +\frac{\gamma \theta_{0}}{m} \frac{\partial^{2}}{\partial \mathbf{v} \partial \mathbf{v}}:\left\langle\sum_{i, j \neq i} w\left(R_{i j}\right) \hat{\mathbf{R}}_{i j} \hat{\mathbf{R}}_{i j} \delta\left(\mathbf{x}-\mathbf{x}_{i}\right)\right\rangle,
\end{aligned}
$$

where $\nabla=\partial / \partial \mathbf{r}$. Performing the integrals over all variables except $\mathbf{x}_{i}$ and $\mathbf{x}_{j}$ leads to

$$
\begin{aligned}
\partial_{t} f+\mathbf{v} \cdot \nabla f= & \gamma \int d \mathbf{v}^{\prime} \int d \mathbf{R} \hat{\mathbf{R}} \hat{\mathbf{R}} w(\mathbf{R}):\left\{\frac{\partial}{\partial \mathbf{v}}\left(\mathbf{v}-\mathbf{v}^{\prime}\right)\right. \\
& \left.+\frac{\theta_{0}}{m} \frac{\partial^{2}}{\partial \mathbf{v} \partial \mathbf{v}}\right\} f^{(2)}\left(\mathbf{v}, \mathbf{r}, \mathbf{v}^{\prime}, \mathbf{r}-\mathbf{R}, t\right) .
\end{aligned}
$$

This is the first equation of the BBGKY hierarchy with the Fokker-Planck equation (4) taking the place of the Liouville equation as the evolution equation. Under the molecular chaos approximation (31) we have the following closed equation for the one-particle distribution function:

$$
\begin{aligned}
\partial_{t} f+\mathbf{v} . \nabla f= & I(f) \equiv \gamma \int d \mathbf{v}^{\prime} \int d \mathbf{R} \hat{\mathbf{R}} \hat{\mathbf{R}} w(R) \\
& \times f\left(\mathbf{v}^{\prime}, \mathbf{r}-\mathbf{R}, t\right):\left\{\frac{\partial}{\partial \mathbf{v}}\left(\mathbf{v}-\mathbf{v}^{\prime}\right)+\frac{\theta_{0}}{m} \frac{\partial^{2}}{\partial \mathbf{v} \partial \mathbf{v}}\right\} \\
& \times f(\mathbf{v}, \mathbf{r}, t) .
\end{aligned}
$$

The molecular chaos approximation is a mean-field approximation, which neglects dynamical correlations resulting from correlated multiple collisions taking place inside an action sphere. As we have set all conservative forces equal to zero, the molecular chaos assumption is exact in the global equilibrium state. Indeed, simulation results show that this is in fact an excellent approximation in the small-time step limit (as shown in Fig. 2).

It can be shown in a similar fashion to Sec. III that the functional

$$
\mathcal{F}=\int d \mathbf{x}\left\{\frac{1}{2} m \mathbf{v}^{2}+\theta_{0} \ln f(\mathbf{x}, t)\right\} f(\mathbf{x}, t)
$$

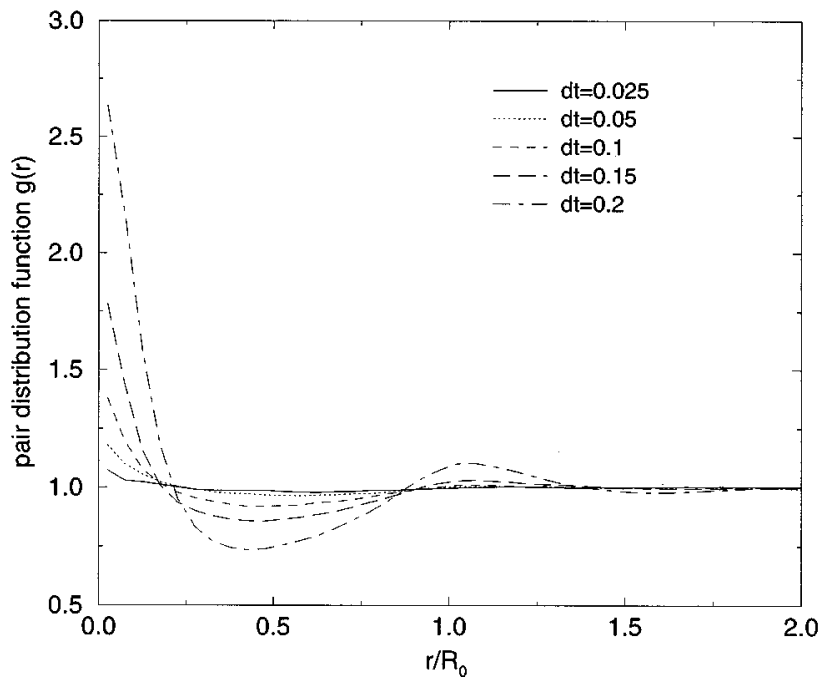

FIG. 2. Two-particle distribution function. The system parameters in the simulation were taken: $N=2000$ particles, friction constant $\gamma=1$, particle density $n=0.2$, action sphere radius $R_{0}=4$, and $w_{D}(r)=2\left(1-r / R_{0}\right)$.

satisfies an $H$ theorem $\left(\partial_{t} \mathcal{F} \leqslant 0\right)$ where the equality only holds if $f(\mathbf{x}, t)$ is given by the equilibrium form $n_{0} \varphi_{0}(\mathbf{v})$ of Eq. (25), which establishes the existence of a unique global equilibrium state.

The next problem is to solve the nonlinear FPB equation, and to analyze the approach to equilibrium using the Chapman-Enskog (CE) method. According to this method, one can distinguish two stages in the evolution of the singleparticle distribution function $f(\mathbf{x}, t)$ : a rapid kinetic stage and a slow hydrodynamic stage [24].

In the kinetic stage, $f(\mathbf{x}, t)$ decays within a characteristic kinetic time $t_{0}$ to the so-called normal solution $f(\mathbf{v} \mid a(\mathbf{r}, t))$ which depends on space and time only through the first few moments $a(\mathbf{r}, t)=\int d \mathbf{v} a(\mathbf{v}) f(\mathbf{x}, t)$, the conserved densities, where $a(\mathbf{v})=\{1, \mathbf{v}, \ldots\}$ are the collisional invariants. In fluid systems the time $t_{0}$ is the mean free time, whereas in a DPD system, $t_{0}$ is estimated from Eq. (36) as $t_{0} \sim 1 /\left(\gamma n R_{0}^{d}\right)$.

In the subsequent hydrodynamic stage, $f$ depends only on space and time through its dependence on the conserved densities. In this stage, the solution $f(\mathbf{v} \mid a(\mathbf{r}, t))$ of the FPB equation can be determined perturbatively, $f=f_{0}+\mu f_{1}+\cdots$, as an expansion in powers of a small parameter, $\mu \sim l_{0} \nabla$, which measures the variation of the macroscopic parameters over a characteristic kinetic length scale, $l_{0} \simeq t_{0} \bar{v}=(1 / \gamma) \sqrt{\theta_{0} / m}$, where $\bar{v}=\sqrt{\theta_{0} / m}$ is a typical mean velocity. Therefore the $\mu$ expansion is essentially an expansion in the small parameter $1 / \gamma$ (cf. solution to Kramers's equation in [25]).

In the remaining part of this section, we focus on determining the lowest order solution $f_{0}$ of Eq. (36), which is the local equilibrium distribution. We first observe that the left hand side of Eq. (36) is of $O(\mu)$, as $\partial_{t} f$ is proportional to $\partial_{t} a \sim O(\mu)$, and similarly for the gradient term. The right hand side of Eq. (36) is of $O(1)$. This requires that, to the dominant order in $\mu, f$ should satisfy $I\left(f_{0}\right)=0+O(\mu)$. To determine the solution $f_{0}$, we delocalize the collision operator $I\left(f_{0}\right)$ by replacing $f_{0}\left(\mathbf{v}^{\prime}, \mathbf{r}-\mathbf{R}, t\right)$ on the right hand side of Eq. (36) by $f_{0}\left(\mathbf{v}^{\prime}, \mathbf{r}, t\right)+O(\mu)$. If we denote the delocal- 
ized collision operator by $I_{0}$, then $f_{0}$ is the solution of

$$
I_{0}\left(f_{0}\right)=0 \text {. }
$$

Guided by the $H$ theorem, we assume the standard form for the local equilibrium distribution:

$$
f_{0}(\mathbf{v} \mid a)=n\left(\frac{m}{2 \pi \theta}\right)^{d / 2} \exp \left[-\frac{m(\mathbf{v}-\mathbf{u})^{2}}{2 \theta}\right],
$$

where $n, \theta$, and $\mathbf{u}$ are arbitrary functions of $\mathbf{r}$ and $t$. Substitution of Eq. (39) into Eq. (38) shows, however, that the above $f_{0}$ is only a solution if

$$
\theta=\theta_{0} \equiv \frac{m \sigma^{2}}{2 \gamma}
$$

where $\theta_{0}$ is the constant model parameter introduced below Eq. (6), which equals the global equilibrium temperature. The parameters $n(\mathbf{r}, t)$ and $\mathbf{u}(\mathbf{r}, t)$ in Eq. (39) are chosen to be the fluid density and flow velocity. Hence,

$$
a(\mathbf{r}, t)=\int d \mathbf{v} a(\mathbf{v}) f(\mathbf{x}, t)=\int d \mathbf{v} a(\mathbf{v}) f_{0}(\mathbf{x}, t),
$$

where $a(\mathbf{v})=\{1, \mathbf{v}\}$ is a collisional invariant.

This observation, Eq. (40), has a profound consequence for the physical processes occurring in the DPD system, and makes it very different from standard fluids with energy conservation. In fluids, there is a fast kinetic relaxation to a local equilibrium state specified by $n(\mathbf{r}, t), \theta(\mathbf{r}, t)$, and $\mathbf{u}(\mathbf{r}, t)$, and a subsequent slow hydrodynamic relaxation of these fields to global equilibrium. The DPD system distinguishes itself from standard fluids in the sense that there is a fast relaxation on a time scale $t_{0}$ to a local equilibrium state, Eqs. (39) and (40), specified by $n(\mathbf{r}, t), \mathbf{u}(\mathbf{r}, t)$, and a spatially uniform and constant temperature $\theta_{0}$. The subsequent slow relaxation involves only the density $n(\mathbf{r}, t)$ and flow velocity $\mathbf{u}(\mathbf{r}, t)$.

Consequently, a DPD system is not able to sustain a temperature gradient on hydrodynamic time scales; there is no heat current proportional to a temperature gradient; and there is no heat conductivity. Thus the DPD system describes a thermostated or isothermal process at a fixed temperature $\theta_{0}$. It may only model physical systems where the temperature either relaxes very rapidly to an equilibrium value or where the temperature is irrelevant (an athermal process). The same conditions of rapid thermal relaxation or athermal processes apply to lattice gas automata [26], where, in the majority of models [27], energy conservation is not satisfied during collisions. There the particles may be considered as hard, impenetrable point particles, as opposed to DPD, where the athermal system consists of completely interpenetrable particles.

It is worthwhile to explore the differences between DPD and standard fluids somewhat further. Recalling that conservative forces have been set equal to zero in the present situation permits us to write the energy density $e(\mathbf{r}, t)$ $=(d / 2) n(\mathbf{r}, t) \theta(\mathbf{r}, t)$ in terms of a kinetic temperature $\theta(\mathbf{r}, t)$. Clearly $n(\mathbf{r}, t)$ is a slowly changing variable, but what is the behavior of $\theta(\mathbf{r}, t)$ ?

To answer this question, it is sufficient to consider only small deviations from global equilibrium, $\delta f=f-n_{0} \varphi_{0}(\mathbf{v})$, and to linearize the FPB equation around $n_{0} \varphi_{0}(\mathbf{v})$. From that equation we shall derive how $\delta \theta(\mathbf{r}, t)=\theta(\mathbf{r}, t)-\theta_{0}$ decays to zero. Linearization of Eq. (36) yields, after some algebra,

$$
\begin{aligned}
\partial_{t} \delta f+\mathbf{v} \cdot \nabla \delta f= & \omega_{0} \frac{\partial}{\partial \mathbf{v}} \cdot\left(\mathbf{v}+\frac{\theta_{0}}{m} \frac{\partial}{\partial \mathbf{v}}\right) \delta f \\
& +\frac{m n_{0}^{2} \gamma}{\theta_{0}} \int d \mathbf{R} w(R) \hat{\mathbf{R}} \hat{\mathbf{R}}: \mathbf{u}(\mathbf{r}-\mathbf{R}) \mathbf{v} \varphi_{0}(\mathbf{v})
\end{aligned}
$$

where we have used the relation

$$
n_{0} \mathbf{u}(\mathbf{r}, t)=\int d \mathbf{v} \delta f(\mathbf{x}, t) \mathbf{v}
$$

and introduced the coefficient $\omega_{0}=1 / t_{0}$,

$$
\omega_{0}=\frac{\gamma n_{0}}{d} \int d \mathbf{R} w(R) \equiv \frac{\gamma n_{0}}{d}[w] .
$$

The equation with its nonlocal integral operator on the right hand side of Eq. (42) would be the starting point for studying generalized hydrodynamics with wave-number-dependent transport coefficients. Here, however, we shall only consider the decay of

$$
\delta \theta(\mathbf{r}, t)=\frac{1}{n_{0}} \int d \mathbf{v}\left(\frac{m \mathbf{v}^{2}}{d}-\theta_{0}\right) \delta f .
$$

Then the rate of change of $\delta f$ can be calculated from Eq. (42), and yields:

$$
\partial_{t} \delta \theta+\nabla \cdot \frac{1}{n_{0}} \int d \mathbf{v}\left(\frac{m \mathbf{v}^{2}}{d}-\theta_{0}\right) \mathbf{v} \delta f=-2 \omega_{0} \delta \theta
$$

The second term on the left hand side of Eq. (46) is typically an $O(\mu)$ correction to the dominant decay terms. So Eq. (46) is a simple relaxation equation which shows explicitly that the kinetic temperature $\theta(\mathbf{r}, t)$ decays within the kinetic stage to the global temperature $\theta_{0}$ with a relaxation time $t_{\theta}=\left(2 \omega_{0}\right)^{-1}=\frac{1}{2} t_{0}$.

The conclusion is that the energy density in the hydrodynamic stage, given by $e(\mathbf{r}, t)=(d / 2) \theta_{0} n(\mathbf{r}, t)$, is still a slow but not an independent variable. It is strictly proportional to the density. Moreover, we can conclude that the free-energytype functions (6) and (37) represent the actual free energy of the DPD system in the hydrodynamic stage.

Similarly, we can determine the local equilibrium part of the pressure tensor (23) in the absence of conservative forces, by replacing $f$ in $\boldsymbol{\Pi}_{K}$ by its local equilibrium form $f_{0}$ and $f^{(2)}$ in $\boldsymbol{\Pi}_{D}$ by $f_{0} f_{0}$ according to the Stosszahlansatz [20]. To zeroth order in $\mu$, the dissipative part $\mathbf{\Pi}_{D}$ vanishes, and the local equilibrium pressure is given by

$$
\mathbf{\Pi}_{0}=\int d \mathbf{v} m \mathbf{V V} f_{0}=n(\mathbf{r}, t) \theta_{0} \mathbb{I}_{\text {. }}
$$

These results will be needed in the next section to solve the FPB equation to linear order in $\mu$. 


\section{HYDRODYNAMIC STAGE}

\section{A. Chapman-Enskog method}

In Sec. IV we have derived the Fokker-Planck-Boltzmann equation for the DPD system and described the ChapmanEnskog method for obtaining its solution $f(\mathbf{v} \mid n, \mathbf{u})$ in the hydrodynamical stage. The method requires that the right and left hand sides of the FPB equation (36) are expanded in powers of $\mu \sim l_{0} \nabla$, using the expansion

$$
f(\mathbf{v} \mid n, \mathbf{u})=f_{0}+\mu f_{1}+\cdots .
$$

Every $\nabla$ is replaced by $\mu \nabla$ and the derivative $\partial_{t} f_{0}$ is eliminated using the macroscopic conservation laws. The lowest order solution, which is the local equilibrium distribution,

$$
f_{0}=n(\mathbf{r}, t)\left(\frac{m}{2 \pi \theta_{0}}\right)^{d / 2} \exp \left[-\frac{m}{2 \theta_{0}}[\mathbf{v}-\mathbf{u}(\mathbf{r}, t)]^{2}\right] \text {, }
$$

has been determined in the preceding section.

To obtain $f_{1}$ we expand the FPB equation in powers of $\mu$, yielding

$$
\frac{\partial f_{0}}{\partial t}+\mu \mathbf{v} \cdot \nabla f_{0}=I\left(f_{0}\right)+\mu(d I / d f)_{f_{0}} f_{1}+\cdots
$$

We start with the right hand side of Eq. (50), which has been calculated exactly to $O(\mu)$ terms included. One finds after some algebra that $I\left(f_{0}\right)=O\left(\mu^{2}\right)$. In the preceiding section it has only been verified that $I\left(f_{0}\right)=O(\mu)$. The latter result is not sufficient, whereas the former is sufficient for our present purpose. In the remaining terms on the right hand side we replace the collision operator $I$ by its delocalized form $I_{0}$, as defined below Eq. (38). The right hand side of Eq. (50) then becomes

$$
(d I / d f)_{f_{0}} f_{1}=\omega_{0} \frac{\partial}{\partial \mathbf{V}} \cdot\left(\mathbf{V}+\frac{\theta_{0}}{m} \frac{\partial}{\partial \mathbf{V}}\right) f_{1},
$$

with $\omega_{0}$ defined in Eq. (44).

To calculate the left hand side of Eq. (50) to $O(\mu)$ we need the rate of change of $n$ and $\mathbf{u}$ to lowest order in $\mu$, which may be calculated from the conservation equations (21) with $\Pi$ replaced by its local equilibrium part $\boldsymbol{\Pi}_{0}=n \theta_{0} \mathbb{I}$, calculated in Eq. (47), i.e.,

$$
\begin{gathered}
\partial_{t} n=-\nabla \cdot(n \mathbf{u}), \\
\partial_{t} \mathbf{u}=-\mathbf{u} \cdot \nabla \mathbf{u}-\frac{\theta_{0}}{\rho} \nabla n .
\end{gathered}
$$

They yield in combination with Eq. (49)

$$
\frac{\partial f_{0}}{\partial t}+\mathbf{v} \cdot \nabla f_{0}=f_{0}[\mathrm{~J}: \mathrm{D}+\mathcal{N} \nabla \cdot \mathbf{u}]
$$

with

$$
\mathrm{J}_{\alpha \beta}(V)=\frac{m}{\theta_{0}}\left\{V_{\alpha} V_{\beta}-\frac{1}{d} \delta_{\alpha \beta} V^{2}\right\},
$$

$$
\mathrm{D}_{\alpha \beta}(V)=\frac{1}{2}\left\{\nabla_{\alpha} u_{\beta}+\nabla_{\beta} u_{\alpha}-\frac{2}{d} \delta_{\alpha \beta} \nabla \cdot \mathbf{u}\right\},
$$

and

$$
\mathcal{J}(V)=\frac{m V^{2}}{d \theta_{0}}-1
$$

Note that the density and temperature gradients are absent on the right hand side of Eq. (53), in contrast to the traditional Chapman-Enskog result [20].

For convenience of notation we introduce the FokkerPlanck operator $\mathcal{L}$ and its adjoint $\mathcal{L}^{+}$, defined as

$$
\begin{gathered}
\mathcal{L}=\frac{\partial}{\partial \mathbf{V}} \cdot\left(\mathbf{V}+\frac{\theta_{0}}{m} \frac{\partial}{\partial \mathbf{V}}\right), \\
\mathcal{L}^{+}=\left(-\mathbf{V}+\frac{\theta_{0}}{m} \frac{\partial}{\partial \mathbf{V}}\right) \cdot \frac{\partial}{\partial \mathbf{V}},
\end{gathered}
$$

to write the final equation for $f_{1}$ as

$$
\omega_{0} \mathcal{L} f_{1}=f_{0}[\mathrm{~J}: \mathrm{D}+\mathcal{J} \nabla \cdot \mathbf{u}]
$$

It is a second order partial differential equation (PDE) with an inhomogeneity on the right hand side. We first construct a special solution by recalling that the Fokker-Planck operator $\mathcal{L}$ can be mapped onto the Schrödinger equation for an isotropic $d$-dimensional harmonic oscillator [25]. Its eigenfunctions are the tensor Hermite polynomials, usually called Sonine polynomials in a kinetic theory context, and the microscopic fluxes $\mathrm{J}$ and $\mathcal{J}$ are among them, i.e.,

$$
\begin{array}{cl}
\mathcal{L} f_{0} \mathrm{~J}=-2 f_{0} \mathrm{~J} ; & \mathcal{L}^{+} \mathrm{J}=-2 \mathrm{~J}, \\
\mathcal{L} f_{0} \mathcal{J}=-2 f_{0} \mathcal{J} ; & \mathcal{L}^{+} \mathcal{J}=-2 \mathcal{J} .
\end{array}
$$

This can easily be verified. Combination of Eqs. (57) and (58) yields the special solution

$$
f_{1}=-\frac{1}{2 \omega_{0}} f_{0}[\mathrm{~J}: \mathrm{D}+\mathcal{J} \cdot \mathbf{u}] .
$$

The general solution is obtained by adding an arbitrary linear combination of collisional invariants $a(\mathbf{V})=\{1, \mathbf{V}\}$, which are the solutions to the homogeneous equation $\mathcal{L} f_{0} a(\mathbf{V})=0$. However, the constraint (41) suppresses these terms and $f_{1}$ is the desired solution of the FPB equation to linear order in $\mu$.

\section{B. Navier-Stokes equation}

The only slow macroscopic fields are the density $n(\mathbf{r}, t)$ and the flow velocity $\mathbf{u}(\mathbf{r}, t)$, leading to the continuity equation and Navier-Stokes equation. The energy density in the hydrodynamic stage, $e(\mathbf{r}, t)=(d / 2) n(\mathbf{r}, t) \theta_{0}$, is not an independent variable. The energy balance equation derived in the Appendix is only relevant in the kinetic stage, but has no physical significance in the hydrodynamical stage.

The results for $f_{0}$ and $f_{1}$ are sufficient to obtain the hydrodynamic equations to Navier-Stokes order and to obtain explicit expressions for the transport coefficients. The 
$O(\mu)$ correction $f_{1}$ contains only gradients of the flow field, $\nabla \mathbf{u}$, but no gradients of the temperature. Therefore there will be no heat current and vanishing heat conductivity. The $O(\mu)$ terms in the pressure tensor $\boldsymbol{\Pi}=\boldsymbol{\Pi}_{0}+\mu \boldsymbol{\Pi}_{1}+\cdots$ will be proportional to $\nabla \mathbf{u}$ and we define the viscosities as the coefficients of proportionality through the constitutive relation

$$
\boldsymbol{\Pi}_{1}=-2 \eta \mathrm{D}-\zeta \nabla \cdot \mathbf{u} \Pi,
$$

where $\eta$ and $\xi$ are, respectively, shear and bulk viscosity.

Combining Eqs. (60), (47), and (21) then yields the Navier-Stokes equation for the DPD system,

$$
\partial_{t}(\rho \mathbf{u})+\nabla \cdot(\rho \mathbf{u u})=-\theta_{0} \nabla n+\nabla \cdot(2 \eta \mathrm{D}+\zeta \nabla \cdot \mathbf{u I}) .
$$

The explicit expressions will be obtained in the next section.

\section{TRANSPORT COEFFICIENTS}

\section{A. Kinematic viscosities $\eta_{K}$ and $\zeta_{K}$}

There are two contributions to the pressure tensor: the kinetic part $\boldsymbol{\Pi}_{K}$ and the dissipative part $\boldsymbol{\Pi}_{D}$, defined in Eq. (23) and two corresponding viscosities. The kinetic part depends only on $f=f_{0}+\mu f_{1}$, which are given in Eqs. (49) and (59). Then $\boldsymbol{\Pi}_{K}$ becomes

$$
\boldsymbol{\Pi}_{K}=n \theta_{0} I+\mu \boldsymbol{\Pi}_{K, 1}+\cdots,
$$

where $\mu$ is a formal expansion parameter that will be set equal to unity at the end of the calculations and

$$
\boldsymbol{\Pi}_{K, 1}=\int d \mathbf{v} m \mathbf{V} \mathbf{V} f_{1}=\theta_{0} \int d \mathbf{v}\{\mathbf{J}(\mathbf{V})+\mathcal{J}(\mathbf{V}) \llbracket\} f_{1} .
$$

Here the dyadic $m \mathbf{V V}$ has been split up into a traceless tensor $\theta_{0} \mathrm{~J}$ and a term $\theta_{0} \mathcal{I}$ proportional to the unit tensor and we have used the relation $\int d \mathbf{v} f_{1}=0$ [see Eq. (41)]. Inserting the explicit solution (59) into Eq. (63) allows us to write Eq. (62) in the form

$$
\Pi_{K, 1}=-\frac{n \theta_{0}}{2 \omega_{0}}\langle\mathrm{~J} \mid \mathrm{J}\rangle: \mathrm{D}-\frac{n \theta_{0}}{2 \omega_{0}}\langle\mathcal{J} \mathcal{J}\rangle \nabla \cdot \mathbf{u} \mathrm{I},
$$

where we have used the relation $f_{0}=n \varphi_{0}(V)$ [see Eq. (25)] and introduced the inner product

$$
\langle A \mid B\rangle=\int d \mathbf{v} \varphi_{0}(V) A(V) B(V) .
$$

Moreover a crossproduct of a traceless tensor and a scalar vanishes, i.e, $\langle\mathcal{J} \mid \mathcal{J}\rangle=0$. The product $\langle\mathcal{J} \mid \mathcal{J}\rangle$ involves a simple Gaussian integral and yields

$$
\langle\mathcal{J} \mid \mathcal{J}\rangle=\frac{2}{d} .
$$

The fourth rank tensor $\langle\mathrm{J} \mid \mathrm{J}\rangle$ is isotropic, traceless, and symmetric, which implies the general form

$$
\begin{aligned}
\left\langle\mathrm{J}_{\alpha \beta} \mid \mathrm{J}_{\delta \gamma}\right\rangle & =\left(\frac{m}{\theta_{0}}\right)^{2} \int d \mathbf{v} \varphi_{0}(V)\left(V_{\alpha} V_{\beta}-\frac{1}{d} \delta_{\alpha \beta} V^{2}\right) V_{\gamma} V_{\delta} \\
& =C\left[\delta_{\alpha \gamma} \delta_{\beta \delta}+\delta_{\alpha \delta} \delta_{\beta \gamma}-\frac{2}{d} \delta_{\alpha \beta} \delta_{\gamma \delta}\right] .
\end{aligned}
$$

By taking double contractions and evaluating Gaussian integrals the constant $C$ comes out to be equal to 1 and the (:) product in Eq. (64) yields

$$
\langle J \mid J\rangle: D=2 D .
$$

Combination of Eqs. (64), (66), and (68) finally yields

$$
\boldsymbol{\Pi}_{K, 1}=-\frac{n \theta_{0}}{\omega_{0}} \mathrm{D}-\frac{n \theta_{0}}{d \omega_{0}} \nabla \cdot \mathbf{u} \mathbf{I} .
$$

Comparison with the constitutive relation (60) enables us to identify the coefficients as the kinetic parts of the viscosities,

$$
\eta_{K}=\frac{n \theta_{0}}{2 \omega_{0}}=\frac{d \theta_{0}}{2[w] \gamma}, \quad \zeta_{K}=\frac{n \theta_{0}}{d \omega_{0}}=\frac{\theta_{0}}{\gamma[w]},
$$

where the definition (44) of $\omega_{0}$ has been used. We note that the kinetic part is inversely proportional to $\gamma$ and has been explicitly calculated.

\section{B. Dissipative viscosities $\eta_{D}$ and $\zeta_{D}$}

This section deals with the dissipative part $\Pi$ of the pressure tensor in Eq. (23), which depends on the pair distribution function $f^{(2)}$. This function has a local equilibrium part $f_{0}^{(2)}$ and a part $\mu f_{1}^{(2)}$, linear in the gradients. We start with the first part.

In order to make a direct comparison with the work of Español [12] we retain the conservative forces, for the time being. Then, the local equilibrium pair function has the form

$$
f_{0}^{(2)}\left(\mathbf{x}, \mathbf{x}^{\prime}\right)=f_{0}(\mathbf{x}) f_{0}\left(\mathbf{x}^{\prime}\right) g_{0}\left(\left|\mathbf{x}-\mathbf{x}^{\prime}\right|\right),
$$

where $g_{0}(R)$ is the spatial correlation function in local equilibrium. Only at the end of the calculation we will set the conservative forces equal to zero, so that $g_{0}(R)=1$.

Substitution of Eq. (71) into Eq. (23) yields then

$$
\begin{aligned}
\Pi_{D}= & -\frac{1}{2} \gamma m \int d \mathbf{R} g_{0}(R) w(R) \hat{\mathbf{R}} \hat{\mathbf{R}}\{\mathbf{R} \cdot[\mathbf{u}(\mathbf{r})-\mathbf{u}(\mathbf{r}-\mathbf{R})]\} \\
& \times n(\mathbf{r}) n(\mathbf{r}-\mathbf{R}) \\
\simeq & -\frac{1}{2} \gamma m n^{2} \int d \mathbf{R} R^{2} g_{0}(R) w(R) \hat{\mathbf{R}} \hat{\mathbf{R}} \hat{\mathbf{R}} \hat{\mathbf{R}}: \nabla \mathbf{u}
\end{aligned}
$$

where $[\cdots]$ has been expanded to linear order in the gradients. Calculation of the completely symmetric isotropic fourth rank tensor proceeds as in Eq. (67) with the result

$$
\begin{aligned}
\int & d \mathbf{R} R^{2} g_{0}(R) w(R) \hat{R}_{\alpha} \hat{R}_{\beta} \hat{R}_{\gamma} \hat{R}_{\delta} \\
= & \frac{\left[R^{2} w g_{0}\right]}{d(d+2)}\left[\delta_{\alpha \beta} \delta_{\gamma \delta}+\delta_{\alpha \delta} \delta_{\beta \gamma}+\delta_{\alpha \gamma} \delta_{\beta \delta}\right],
\end{aligned}
$$




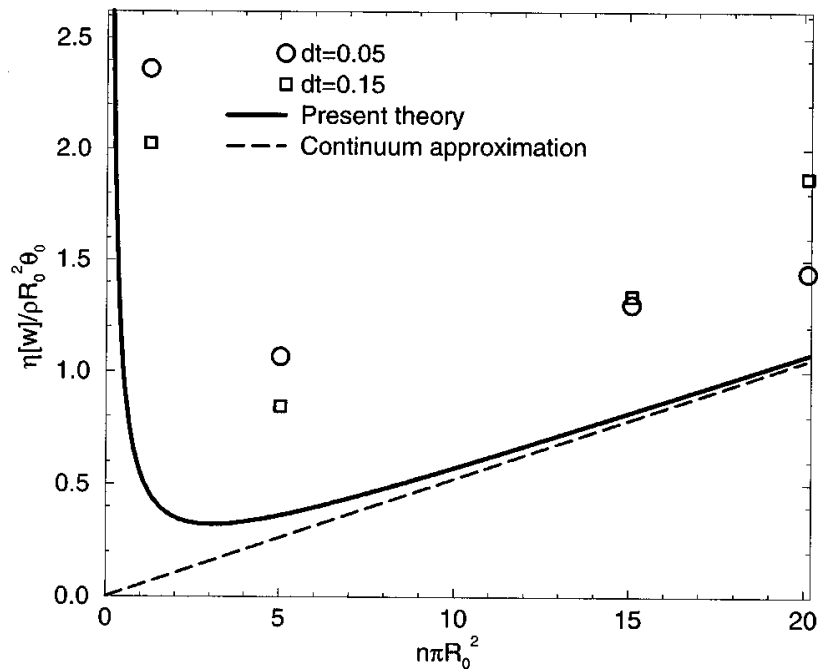

FIG. 3. Kinematic viscosity $\nu=\eta / \rho$ against density $n$ for $d t=0.05$ and $d t=0.15$ in dimensionless units. The system parameters in the simulations were taken: friction constant $\gamma=1$ and random force strength $\sigma=1.5$ for densities $n=0.025,0.1,0.3$, and 0.4 . Lines indicate present theory and results as obtained by Hoogerbrugge and Koelman, and [13], [11] under the continuum approximation.

where

$$
\left[R^{2} w g_{0}\right] \equiv \int d \mathbf{R} R^{2} g_{0}(R) w(R)
$$

We note that the definition of $\boldsymbol{\Pi}_{D}$ in Eq. (23) contains $\bar{f}^{(2)}$ rather than $f^{(2)}\left(\mathbf{v}, \mathbf{r}, \mathbf{v}^{\prime}, \mathbf{r}-\mathbf{R}, t\right)$. One easily verifies that the spatial averaging, denoted by the overline, makes no difference to linear order in the gradients. The final result for the dissipative part then becomes

$$
\mathbf{\Pi}_{D}=-\frac{m \gamma n^{2}\left[R^{2} w g_{0}\right]}{d(d+2)} \mathrm{D}-\frac{m \gamma n^{2}\left[R^{2} w g_{0}\right]}{2 d^{2}} \nabla \cdot \mathbf{u} \mathbb{} .
$$

With the help of Eq. (60) the coefficients can be identified as the contributions to the viscosities due to the dissipative forces, i.e.,

$$
\eta_{D}=\frac{m \gamma n^{2}\left[R^{2} w g_{0}\right]}{2 d(d+2)}, \quad \zeta_{D}=\frac{m \gamma n^{2}\left[R^{2} w g_{0}\right]}{2 d^{2}} .
$$

The local equilibrium contribution (75) to the dissipative pressure tensor turns out to be the dominant contribution to the viscosity of a DPD fluid, for large values of $n \gamma$, as illustrated in Figure 3 and confirmed by numerical simulation in $[4,13]$. We also want to point out that an $O(\mu)$ contribution to the pressure tensor, calculated in local equilibrium as in Eq. (72), is not a novelty of this paper, but also occurs in all systems with impulsive (hard core) interactions that are not strictly local. For instance, consider the collisional transfer contribution analogous to $\boldsymbol{\Pi}_{C}$ for elastic hard spheres, where $\mathbf{F}=-\nabla \phi$ in Eq. (23) is ill defined. This term is calculated in Secs. 16.4 and 16.5 of [20], where its local equilibrium contribution yields $\eta_{\mathrm{HS}}=\frac{3}{5} \zeta_{\mathrm{HS}}=\frac{3}{5} \varpi$ with $\varpi \sim n^{2}$, defined in Eq. (16.5.7) of [20]. These contributions in real fluids are the direct counterparts of $\eta_{D}=\frac{3}{5} \zeta_{D} \sim n^{2}$ in DPD.
We return to the DPD system without conservative forces, where the Gibbs distribution (15) reduces to $\prod_{i=1}^{N} \varphi_{0}\left(v_{i}\right)$, and where the spatial correlations are absent, i.e., $g_{0}(R)=1$. This equality is also required here for consistency with the molecular chaos approximation (31), used in Sec. V and subsequent ones.

So far, we have only considered local equilibrium contributions to $\boldsymbol{\Pi}_{D}$ in Eq. (72). To obtain the complete contribution, consistent with the molecular chaos assumption, we substitute Eq. (31) into Eq. (23) and use the definitions (19). Surprisingly, the results (72) are recovered, showing that Eqs. (72)-(76) give the full contribution of $\boldsymbol{\Pi}_{D}$ to the Navier-Stokes equation, at least within the molecular chaos assumption.

To facilitate the comparison with the original predictions of $[4,13,11]$, we set $g_{0}=1$ in Eq. (75) and introduce

$$
\left\langle R^{2}\right\rangle_{w}=\left[R^{2} w\right] /[w]
$$

where $[a]$ denotes the spatial average introduced in Eq. (44), so that $\left\langle R^{2}\right\rangle_{w} \sim R_{0}^{2}$.

The final result for the dissipative part of the viscosities is then

$$
\begin{gathered}
\eta_{D}=\frac{\gamma m n^{2}\left\langle R^{2}\right\rangle_{w}[w]}{2 d(d+2)}=\omega_{0} t_{w}^{2} n \theta_{0} / 2(d+2), \\
\zeta_{D}=\frac{\gamma m n^{2}\left\langle R^{2}\right\rangle_{w}[w]}{2 d^{2}}=\omega_{0} t_{w}^{2} n \theta_{0} / 2 d,
\end{gathered}
$$

where $\omega_{0}=1 / t_{0}=\gamma n[w] / d$ is the characteristic relaxation rate introduced in Eq. (44), and $t_{w}$, defined through $t_{w}^{2}=\left\langle R^{2}\right\rangle_{w} \bar{v}^{2}$, is the average traversal time of an action sphere with $\bar{v}=\left(\theta_{0} / m\right)^{1 / 2}$ the thermal velocity. These results are in fact the theoretical predictions for the total shear and bulk viscosity of the DPD fluid, as obtained in $[13,11]$ on the basis of the "continuum approximation" to the equations of motion of the DPD particles. In the present context of nonequilibrium statistical mechanics and kinetic theory, these contributions have been identified as the local equilibrium contributions to the transport coefficients in order to make the connection with Hoogerbrugge and Koelman's expression for the kinematic viscosity:

$$
\nu=\eta / \rho=\frac{\omega\left\langle R^{2}\right\rangle_{w}}{2 d(d+2) \delta t},
$$

with their friction constant $\omega=\gamma \delta t$, proportional to $\delta t$, as a proper friction should be. Moreover, we recall that the range function $w(R)$ in [4] is normalized as

$$
n[w]=n \int d \mathbf{R} w(R)=1 .
$$

So, the results (78) and (79) are identical. Hoogerbrugge and Koelman have also shown that the viscosity found in their numerical simulations approaches Eqs. (78) and (79) for large $n \gamma$. Simulations carried out with the modified DPD algorithm show the same properties [13]. 
We conclude this subsection by listing the full results (70) and (78) for the shear and bulk viscosity in a DPD fluid with continuous time $(\delta t \rightarrow 0)$ :

$$
\begin{aligned}
& \eta=\eta_{D}+\eta_{K}=\frac{1}{2} n \theta_{0}\left\{\frac{\omega_{0} t_{w}^{2}}{d+2}+\frac{1}{\omega_{0}}\right\}, \\
& \zeta=\zeta_{D}+\zeta_{K}=\frac{1}{d} n \theta_{0}\left\{\frac{\omega_{0} t_{w}^{2}}{2}+\frac{1}{\omega_{0}}\right\} .
\end{aligned}
$$

They involve the two intrinsic time scales of the DPD fluid: the characteristic kinetic time $t_{0}=1 / \omega_{0}$ [see Eq. (44)] and the traversal time $t_{w}$ of an action sphere, as defined below Eq. (78), which is of order $R_{0} / \bar{v}$.

In the parameter range $t_{w}>t_{0}$ the estimates $\eta_{D}$ and $\zeta_{D}$ of $[13,11]$ dominate, and in the range $t_{w}<t_{0}$ the kinematic viscosities do, as illustrated in Fig. 3.

\section{Numerical simulations}

As a simple test, the shear viscosity $\eta$ of the DPD system was measured in two dimensions using a physical method. A linear velocity gradient was established between two moving plates and the force required to maintain this system was measured once equilibrium had been attained.

By means of these simulations, we have measured the viscosity of the DPD fluid as a function of $n \gamma$ at different temperatures $\theta_{0}=m \sigma^{2} / 2 \gamma$. Results are shown in Fig. 3 for a higher temperature to emphasize the importance of the kinematic contribution. At large $n \gamma$ the measured viscosity approaches the theoretical prediction when the time step $\delta t$ is reduced. In this range of parameters, the viscosity is dominated by its dissipative part (78), corresponding to the original estimates of Hoogerbrugge and Koelman. At small $n \gamma$ and high temperature $\theta_{0}$ the viscosity is dominated by the kinetic contribution.

At small $n \gamma$ there are sizable differences between predicted and simulated results, which do not decrease with decreasing time step size. The breakdown of the theory in this range of parameters could be explained by several factors.

(1) First, inspection of the collision term on the right hand side of Eq. (36) or Eq. (51) shows that with $\omega_{0} \sim n \gamma$ and $\omega_{0} \theta_{0} \sim \sigma^{2}$ small the typical size of the collision term $\sim 1 / t_{0}$ may not be large compared to the propagation terms on the right hand side of Eq. (36). Consequently, the ChapmanEnskog expansion will be poorly convergent or even divergent, because the kinetic and hydrodynamic time regimes are no longer well separated, or, equivalently, because the change of the macroscopic flow velocity over the characteristic kinetic length scale becomes large. To be consistent with the physical requirement of well separated time scales in this range of parameters, the imposed velocity gradients would have to be reduced.

(2) The system size for the simulations carried out may be too small. In order to avoid finite-size effects such as wavevector-dependent viscosities, the system must be significantly larger than the interaction range $R_{0}$ of the particles.

(3) The molecular chaos approximation (31) could break down as a result of the small net momentum transfer in DPD collisions. This may cause the development of dynamic correlations such as correlated binary ("ring') collisions.

\section{Self-diffusion coefficient $D$}

The coefficient of self-diffusion can be obtained by considering a DPD fluid that is in equilibrium, except for the probability distribution $f_{s}$ of a tagged particle, labeled as $i=1$. Following the arguments of Sec. $\mathrm{V}$ and choosing the $\mu$-space density $\hat{f}_{s}(\mathbf{x})=\delta\left(\mathbf{x}-\mathbf{x}_{1}\right)$, instead of Eq. (32), one arrives at an equation similar to Eq. (35), with $f$ and $f^{(2)}$ replaced by $f_{s}$ and $f_{s}^{(2)}$, respectively, defined as

$$
\begin{gathered}
f_{s}(\mathbf{x}, t)=\left\langle\delta\left(\mathbf{x}-\mathbf{x}_{1}\right)\right\rangle, \\
f_{s}^{(2)}\left(\mathbf{x}, \mathbf{x}^{\prime}, t\right)=\left\langle\sum_{j \neq 1} \delta\left(\mathbf{x}-\mathbf{x}_{1}\right) \delta\left(\mathbf{x}-\mathbf{x}_{j}\right)\right\rangle .
\end{gathered}
$$

The molecular chaos assumption (31) now takes the form

$$
f_{s}^{(2)}\left(\mathbf{x}, \mathbf{x}^{\prime}, t\right)=n \varphi_{0}\left(v^{\prime}\right) f_{s}(\mathbf{x}, t),
$$

as the fluid particles are in thermal equilibrium with the Maxwellian $\varphi_{0}(v)$ defined in Eq. (25), and the resulting FPB equation is linear, i.e.,

$$
\partial_{t} f_{s}+\mathbf{v} \cdot \nabla f_{s}=\omega_{0} \frac{\partial}{\partial \mathbf{v}} \cdot\left(\mathbf{v}+\frac{\theta_{0}}{m} \frac{\partial}{\partial \mathbf{v}}\right) f_{s} .
$$

It is identical to the Kramers equation (1) with $\mathbf{F}(\mathbf{r})=0$.

The continuity equation takes the form

$$
\partial_{t} c(\mathbf{r}, t)+\nabla \cdot \mathbf{j}(\mathbf{r}, t)=0
$$

with tagged particle density and current defined as

$$
\begin{gathered}
c(\mathbf{r}, t)=\int d \mathbf{v} f_{s}(\mathbf{x}, t), \\
\mathbf{j}(\mathbf{v}, t)=\int d \mathbf{v} \mathbf{v} f_{s}(\mathbf{x}, t) .
\end{gathered}
$$

Application of the Chapman-Enskog method to Eq. (84) yields the "local equilibrium" distribution function $f_{s 0}=c(\mathbf{r}, t) \varphi_{0}(v)$ and following equation for $f_{s 1} \equiv f_{s}-f_{s 0}$,

$$
\varphi_{0}(v) \mathbf{v} \cdot \nabla c=\omega_{0} \frac{\partial}{\partial \mathbf{v}} \cdot\left(\mathbf{v}+\frac{\theta_{0}}{m} \frac{\partial}{\partial \mathbf{v}}\right) f_{s 1} \equiv \omega_{0} \mathcal{L} f_{s 1} .
$$

As $\mathbf{v} \phi_{0}$ on the left hand side is again an eigenfunction of $\mathcal{L}$ with eigenvalue -1 , we find

$$
f_{s 1}=-\frac{1}{\omega_{0}} \phi_{0}(v) \mathbf{v} \cdot \nabla c
$$

The coefficient of self-diffusion $D$, defined through the constitutive equation

$$
\mathbf{j}=\int d \mathbf{v} \mathbf{v} f_{1}=-D \nabla c,
$$

becomes for the DPD fluid

$$
D=\frac{\theta_{0}}{\omega_{0} m}=\frac{d \theta_{0}}{\rho \gamma[w]},
$$


where $\rho=m n$ is the mass density of the fluid. The above result is new. There is only a kinetic contribution and no dissipative one.

\section{CONCLUSIONS AND PROSPECTS}

The main results of this paper are the derivation and solution of the Fokker-Planck-Boltzmann equation for the DPD fluid, providing explicit results for the thermodynamic and transport properties in terms of the system parameters: density $n$, friction constant $\gamma$, temperature $\theta_{0}=m \sigma^{2} / 2 \gamma$, and range function $w(R)$ with range $R_{0}$. There are two intrinsic time scales: the kinetic relaxation time $t_{0} \sim 1 / n \gamma R_{0}^{d}$ determined by the collision term, and the traversal time $t_{w} \sim R_{0} / \bar{v}$ of an action sphere, where $\bar{v}=\sqrt{\theta_{0} / m}$ is the average velocity. We highlight the most important results and future prospects in a number of comments.

(1) The DPD fluid for continuous time (step size $\delta t \rightarrow 0$ ), described by the $N$-particle Fokker-Planck equation of Español and Warren, obeys an $H$ theorem for the free energy $\mathcal{F}$. The indispensable role of the detailed balance condition in establishing such a theorem is demonstrated. It guarantees a monotonic approach of $\mathcal{F}$ towards a unique thermal equilibrium, described by the Gibbs distribution with a temperature $\theta_{0}=m \sigma^{2} / 2 \gamma$.

(2) The local conservation laws for mass density $\rho=n m$ and momentum density are the essential prerequisites for the validity of the Navier-Stokes equations. The temperature, however, plays a very peculiar role. On the one hand the detailed balance condition guarantees the existence of a welldefined thermal equilibrium with a global equilibrium $\theta_{0}$, in which energy is conserved on average. On the other hand, the local equilibrium state depends only on $n(\mathbf{r}, t)$ and $\mathbf{u}(\mathbf{r}, t)$, but not on a local equilibrium temperature $\theta(\mathbf{r}, t)$, which relaxes in a time $t_{0}$ (kinetic stage) towards its uniform equilibrium value $\theta_{0}$. In the subsequent hydrodynamic stage the DPD fluid is not able to sustain a temperature gradient, there is no heat conduction, and all processes occur isothermally.

(3) In the coarse-grained mesoscopic interpretation of DPD particles as "lumps of fluids," the microscopic conservative forces between the DPD particles are small compared to the mesoscopic friction and random noise (large $\gamma$ limit), and have been neglected in deriving the FPB equation. At sufficiently low temperature conservative forces can have the effect of forcing the DPD particles into crystalline configurations.

(4) The FPB equation is derived from the first equation of the BBGKY hierarchy for the distribution functions, obtained from the $N$-particle Fokker-Planck equation, playing the role of the Liouville equation. In addition, the molecular chaos assumption $f^{(2)}\left(\mathbf{x}, \mathbf{x}^{\prime}\right)=f(\mathbf{x}) f\left(\mathbf{x}^{\prime}\right)$ has been used.

(5) The Chapman-Enskog solution to the continuous time FPB equation yields two types of contributions to the viscosities. (i) Dissipative parts $\eta_{D}$ and $\zeta_{D}$, accounting for the collisional transfer through the nonlocal dissipative interactions. They are determined by the local equilibrium distribution. (ii) Kinetic parts $\eta_{K}$ and $\zeta_{K}$, coming from the collision operator and determined by the Chapman-Enskog solution of the FPB equation. If $t_{w}>t_{0}$ the dissipative viscosities are dominant; if $t_{w}<t_{0}$ the kinetic viscosities are dominant. In
$[13,11]$ the total viscosity is estimated by $\eta_{D}$ and $\zeta_{D}$, which is correct for $t_{w} \gg t_{0}$. The simulated results for the kinematic viscosity are in reasonably good agreement with the predictions within the assessed theoretical regions of validity of the theory. We also calculated the coefficient of self-diffusion, which only has a kinetic part.

(6) It is also of interest to consider the Green-Kubo formulas for the viscosities in a DPD fluid as derived in [12], where the linear $\gamma$ dependence of the viscosity in the limit of large dissipation $\gamma$ is questioned. To make the connection we observe that $\eta_{D}$ and $\eta_{C}$ of [12] should be identified, respectively, with $\eta_{D}$ and $\eta_{K}$ of the present paper. The time correlation functions of [12] for $\eta_{D}$ and $\eta_{C}$ are formally proportional to $\gamma^{2}$ and 1, respectively. Both time integrals appearing in the Green-Kubo formulas extend over the characteristic kinetic time to $\sim 1 / \gamma$. Consequently, $\eta_{D} \sim \gamma$ and $\eta_{C} \sim 1 / \gamma$ in the limit of large $\gamma$, in complete agreement with the detailed calculation of the present paper.

(7) The validity of the kinetic transport coefficients $\eta_{K}$ and $\zeta_{K}$ and the convergence of the Chapman-Enskog expansion require that spatial variations $\left(\mu \sim l_{0} \nabla\right)$ are small over a characteristic kinetic length scale $l_{0} \sim \bar{v} t_{0} \sim \bar{v} / n \gamma R_{0}^{d}$. The convergence of the gradient expansion in Eq. (72) and the validity of the dissipative viscosities $\eta_{D}$ and $\zeta_{D}$ require in addition that spatial variations are small over the diameter of an action sphere, $R_{0} \sim \bar{v} t_{w}$. Both criteria pose bounds on the shear rates, imposed in the simulations, as well as on the validity of the Chapman-Enskog expansion.

(8) An interesting extension of the present theory would be towards generalized hydrodynamics. Such a region exists if $t_{w} \gg t_{0}$ or $R_{0} \gg l_{0}$. Then, the hydrodynamic modes with wave numbers $k$ in the range $\left(2 \pi / R_{0}, 2 \pi / l_{0}\right)$ have $k$-dependent dissipative viscosities $\eta_{D}$ and $\zeta_{D}$. They may be calculated by studying the eigenmodes of the linearized FPB equation (42). A similar wave vector range to generalized hydrodynamics occurs in dense hard sphere fluids, where $l_{0}$ is small compared to the hard sphere diameter $R_{0}$. Such theories have been used successfully to describe neutron scattering experiments on liquid argon and liquid sodium [28]. Generalized hydrodynamics in DPD might therefore be of interest in explaining light and neutron experiments on concentrated colloidal suspensions.

(9) The equilibrium properties (see Fig. 2 and [14]) and transport coefficients of DPD (see $[16,18]$ ) depend sensitively on the step size $\delta t$. The Fokker-Planck equation (4), the detailed balance condition (16), the FPB equation (36), the hydrodynamic equations (61) and corresponding transport coeffcients in Secs. VII A, VII B, and VII C only hold for the continuous time model $(\delta t \rightarrow 0)$. The only analytic study, available on DPD at finite $\delta t$ [14], calculates the equilibrium temperature $\theta(\delta t)$, and derives criteria, imposed on $\delta t$, for the stability of the equilibrium distribution $f_{0}(\mathbf{x})$.

The most important open problem on DPD is a systematic analysis of all $\delta t$ corrections to equilibrium and transport properties, such as an explanation of Figs. 2 and 3, suggesting that the current form of the modified DPD algorithm for finite step size $\delta t$ does not obey the detailed balance conditions, which implies that its stationary state is not the thermal equilibrium state described by the Gibbs distribution. 


\section{ACKNOWLEDGMENTS}

C.M. acknowledges financial support from EPSRC (UK), ERASMUS (EU), and Unilever Research (UK) and the hospitality of Universiteit Utrecht where most of this work was carried out. G.B. acknowledges the financial support of the foundation "Fundamental Onderzoek der Materie", (FOM), which is financially supported by the Dutch National Science Foundation (NWO). We would also like to thank P. Español, D. Ten Bosch, J. Van den Berg, N. Van Kampen, T. van Noije, P. Warren, and J. Yeomans for helpful discussions and correspondence.

\section{APPENDIX: DETAILS OF THE MACROSCOPIC FLOW EQUATIONS}

\section{Momentum conservation equation}

Inserting $A=\sum_{i} m \mathbf{v}_{i} \delta\left(\mathbf{r}-\mathbf{r}_{i}\right)$ in Eq. (18) yields directly

$$
\begin{aligned}
\partial_{t}(\rho \mathbf{u})= & -\nabla \cdot\left\langle\sum_{i} m \mathbf{v}_{i} \mathbf{v}_{i} \delta\left(\mathbf{r}-\mathbf{r}_{i}\right)\right\rangle+\left\langle\sum_{i} \mathbf{F}_{i} \delta\left(\mathbf{r}-\mathbf{r}_{i}\right)\right\rangle \\
& -m \gamma\left\langle\sum_{i, j \neq i} w\left(R_{i j}\right)\left(\hat{\mathbf{R}}_{i j} \cdot \mathbf{v}_{i j}\right) \hat{\mathbf{R}}_{i j} \delta\left(\mathbf{r}-\mathbf{r}_{i}\right)\right\rangle .
\end{aligned}
$$

The first term on the right hand side, which will be called rhs1, is transformed to the local rest frame of the fluid by introducing peculiar velocities $\mathbf{V}_{i}=\mathbf{v}_{i}-\mathbf{u}\left(\mathbf{r}_{i}, t\right)$ and yields

$$
\operatorname{rhs} 1=-\nabla \cdot\left(\rho \mathbf{u u}+\mathbf{\Pi}_{K}\right),
$$

where

$$
\boldsymbol{\Pi}_{K}=\left\langle\sum_{i} m \mathbf{V}_{i} \mathbf{V}_{i} \delta\left(\mathbf{r}-\mathbf{r}_{i}\right)\right\rangle
$$

is the kinetic part of the pressure tensor, as listed in Eq. (23). The second term on the right hand side of Eq. (A1), which will be called rhs2, involves the conservative interparticle forces $\mathbf{F}_{i}=\sum_{j \neq i} \mathbf{F}\left(\mathbf{R}_{i j}\right)$. Symmetrizing over $i$ and $j$ yields then

$$
\begin{aligned}
\mathrm{rhs} 2 & =\left\langle\frac{1}{2} \sum_{i, j \neq i} \mathbf{F}\left(\mathbf{R}_{i j}\right)\left[\delta\left(\mathbf{r}-\mathbf{r}_{i}\right)-\delta\left(\mathbf{r}-\mathbf{r}_{j}\right)\right]\right\rangle \\
& =-\nabla \cdot\left\langle\frac{1}{2} \sum_{i, j \neq i} \mathbf{F}\left(\mathbf{R}_{i j}\right) \mathbf{R}_{i j} \int_{0}^{1} d \lambda \delta\left(\mathbf{r}-\mathbf{r}_{i}+\lambda \mathbf{R}_{i j}\right)\right\rangle \\
& \equiv-\nabla \cdot \mathbf{\Pi}_{C} .
\end{aligned}
$$

Here we have used the identity

$$
\begin{aligned}
\delta\left(\mathbf{r}-\mathbf{r}_{i}\right)-\delta\left(\mathbf{r}-\mathbf{r}_{j}\right) & =-\int_{0}^{1} d \lambda \frac{d}{d \lambda} \delta\left(\mathbf{r}-\mathbf{r}_{i}+\lambda \mathbf{R}_{i j}\right) \\
& =-\nabla \cdot \mathbf{R}_{i j} \int_{0}^{1} d \lambda \delta\left(\mathbf{r}-\mathbf{r}_{i}+\lambda \mathbf{R}_{i j}\right) .
\end{aligned}
$$

The third term on the right hand side of Eq. (A1), referred to as rhs3, is due to dissipative particle interactions and can be treated in a similar fashion. Symmetrizing over $i$ and $j$, and replacing $\delta\left(\mathbf{r}-\mathbf{r}_{i}\right)$ by $(1 / 2)\left[\delta\left(\mathbf{r}-\mathbf{r}_{i}\right)-\delta\left(\mathbf{r}-\mathbf{r}_{j}\right)\right]$, we obtain

$$
\begin{aligned}
\operatorname{rhs} 3= & \nabla \cdot\left\langle\frac{m}{2} \sum_{i, j \neq i} \gamma w\left(R_{i j}\right) \mathbf{R}_{i j} \hat{\mathbf{R}}_{i j}\left(\hat{\mathbf{R}}_{i j} \cdot \mathbf{v}_{i j}\right)\right. \\
& \left.\times \int_{0}^{1} d \lambda \delta\left(\mathbf{r}-\mathbf{r}_{i}+\lambda \mathbf{R}_{i j}\right)\right\rangle \\
\equiv & -\nabla \cdot \mathbf{\Pi}_{D} .
\end{aligned}
$$

The results (A4) and (A6) are of the same general form, and can be expressed using the pair distribution function (20) as

$$
\begin{aligned}
\left\langle\sum_{i, j \neq i} A\left(\mathbf{R}_{i j}, \mathbf{v}_{i}, \mathbf{v}_{j}\right) \delta\left(\mathbf{r}-\mathbf{r}_{i}+\lambda \mathbf{R}_{i j}\right)\right\rangle \\
=\int d \mathbf{v} \int d \mathbf{v}^{\prime} \int d \mathbf{R} A\left(\mathbf{R}, \mathbf{v}, \mathbf{v}^{\prime}\right) \\
\quad \times f^{(2)}\left(\mathbf{v}, \mathbf{r}+\lambda \mathbf{R}, \mathbf{v}^{\prime}, \mathbf{r}+(\lambda-1) \mathbf{R}, t\right),
\end{aligned}
$$

where Eqs. (A4), (A6), and (A7) yield, respectively, $\boldsymbol{\Pi}_{C}$ and $\boldsymbol{\Pi}_{D}$ as listed in Eq. (23) with $\bar{f}^{(2)}$ defined in Eq. (24). Combination of Eqs. (A1), (A2), (A4), and (A6) gives the macroscopic equation for the momentum density,

$$
\partial_{t}(\boldsymbol{\rho u})+\nabla \cdot\left\{\boldsymbol{\rho u u}+\boldsymbol{\Pi}_{K}+\boldsymbol{\Pi}_{C}+\boldsymbol{\Pi}_{D}\right\}=0,
$$

as listed in Eqs. (21) and (22) in the body of the paper.

\section{Energy balance equation}

We start with the kinetic energy density $e_{K}$ by setting $A=\Sigma_{i}(1 / 2) m \mathbf{v}_{i}^{2} \delta\left(\mathbf{r}-\mathbf{r}_{i}\right)$ in Eq. (18). This yields, after some algebra,

$$
\begin{aligned}
\partial_{t} e_{K}= & -\nabla \cdot\left\langle\sum_{i} \frac{1}{2} m \mathbf{v}_{i}^{2} \delta\left(\mathbf{r}-\mathbf{r}_{i}\right)\right\rangle \\
& +\left\langle\sum_{i, j \neq i} \mathbf{v}_{i} \cdot \mathbf{F}\left(\mathbf{R}_{i j}\right) \delta\left(\mathbf{r}-\mathbf{r}_{i}\right)\right\rangle \\
& -m \gamma\left\langle\sum_{i, j \neq i} w\left(R_{i j}\right)\left(\hat{\mathbf{R}}_{i j} \cdot \mathbf{v}_{i j}\right)\left(\hat{\mathbf{R}}_{i j} \cdot \mathbf{v}_{i}\right) \delta\left(\mathbf{r}-\mathbf{r}_{i}\right)\right\rangle \\
& +\gamma \theta_{0}\left\langle\sum_{i, j \neq i} w\left(R_{i j}\right) \delta\left(\mathbf{r}-\mathbf{r}_{i}\right)\right\rangle .
\end{aligned}
$$

By setting $A=\frac{1}{2} \sum_{i, j \neq i} \phi\left(R_{i j}\right) \delta\left(\mathbf{r}-\mathbf{r}_{i}\right)$ we find similarly for the potential energy density,

$$
\begin{aligned}
\partial_{t} e_{\phi}= & -\nabla \cdot \frac{1}{2}\left\langle\sum_{i, j \neq i} \mathbf{v}_{i} \phi\left(R_{i j}\right) \delta\left(\mathbf{r}-\mathbf{r}_{i}\right)\right\rangle \\
& -\frac{1}{2}\left\langle\sum_{i, j \neq i} \mathbf{v}_{i j} \cdot \mathbf{F}\left(\mathbf{R}_{i j}\right) \delta\left(\mathbf{r}-\mathbf{r}_{i}\right)\right\rangle .
\end{aligned}
$$

We sum Eqs. (A9) and (A10) to obtain the rate of change of the total energy density: 


$$
e=e_{K}+e_{\phi}=\left\langle\sum_{i} \epsilon_{i}(\mathbf{v}) \delta\left(\mathbf{r}-\mathbf{r}_{i}\right)\right\rangle,
$$

where $\epsilon_{i}(\mathbf{v})$ is the microscopic energy per particle:

$$
\epsilon_{i}(\mathbf{v})=\frac{1}{2} m \mathbf{v}_{i}^{2}+\frac{1}{2} \sum_{j \neq i} \phi\left(R_{i j}\right) .
$$

We denote the $n$th term on the right hand sides of Eqs. (A9) and (A10) by (an) and (bn), respectively, and get the following results:

$$
\begin{aligned}
(a 1)+(b 1)= & -\nabla \cdot\left\langle\sum_{i} \mathbf{v}_{i} \epsilon_{i}(\mathbf{v}) \delta\left(\mathbf{r}-\mathbf{r}_{i}\right)\right\rangle \equiv-\nabla \cdot \mathbf{q}_{K}, \\
(a 2)+(b 2)= & \frac{1}{2}\left\langle\sum_{i}\left(\mathbf{v}_{i}+\mathbf{v}_{j}\right) \cdot \mathbf{F}\left(\mathbf{R}_{i j}\right) \delta\left(\mathbf{r}-\mathbf{r}_{i}\right)\right\rangle \\
= & -\nabla \cdot\left\langle\frac{1}{4} \sum_{i, j \neq i} \mathbf{R}_{i j} \mathbf{F}\left(\mathbf{R}_{i j}\right) \cdot\left(\mathbf{v}_{i}+\mathbf{v}_{j}\right)\right. \\
& \left.\times \int_{0}^{1} d \lambda \delta\left(\mathbf{r}-\mathbf{r}_{i}+\lambda \mathbf{R}_{i j}\right)\right\rangle=-\nabla \cdot \mathbf{q}_{C} .
\end{aligned}
$$

The expression for $[(a 2)+(b 2)]$ has been symmetrized over $i$ and $j$ and Eq. (A5) has been used. The term (a4) represents the energy source $\Gamma_{R}$ caused by the random forces. In (a3) we split $\mathbf{v}_{i}$ into $(1 / 2) \mathbf{v}_{i j}+(1 / 2)\left(\mathbf{v}_{i}+\mathbf{v}_{j}\right)$. The first term containing $\mathbf{v}_{i j}$ gives the energy sink $\Gamma_{D}$ resulting from the damping forces. The second term containing $\mathbf{v}_{i j}$ is again symmetrized and combined with Eq. (A5) to give the dissipative part of the energy current $-\nabla \cdot \mathbf{q}_{D}$. Combination of these terms then gives

$$
\begin{aligned}
(a 4)+(a 3)= & \gamma\left\langle\sum_{i, j \neq i} w\left(R_{i j}\right) \delta\left(\mathbf{r}-\mathbf{r}_{i}\right)\right\rangle \\
& -\frac{1}{2} m \gamma\left(\sum_{i, j \neq i} w\left(R_{i j}\right)\left(\hat{\mathbf{R}}_{i j} \cdot \mathbf{v}_{i j}\right)^{2} \delta\left(\mathbf{r}-\mathbf{r}_{i}\right)\right\rangle \\
& +\nabla \cdot\left\langle\frac{1}{4} \sum_{i, j \neq i} \mathbf{R}_{i j} w\left(R_{i j}\right)\left(\hat{\mathbf{R}}_{i j} \cdot \mathbf{v}_{i j}\right) \hat{\mathbf{R}}_{i j} \cdot\left(\mathbf{v}_{i}+\mathbf{v}_{j}\right)\right. \\
& \left.\times \int_{0}^{1} d \lambda \delta\left(\mathbf{r}-\mathbf{r}_{i}+\lambda \mathbf{R}_{i j}\right)\right\rangle \\
& +\gamma\left(\sum_{i, j \neq i} w\left(R_{i j}\right) \delta\left(\mathbf{r}-\mathbf{r}_{i}\right)\right\rangle \\
\equiv & \Gamma_{R}-\Gamma_{D}-\nabla \cdot \mathbf{q}_{D},
\end{aligned}
$$

where $\Gamma_{R}, \Gamma_{D}$, and $\mathbf{q}_{D}$ are defined by the three preceding terms, respectively.

To obtain the full energy balance equation we sum Eqs. (A11) -(A14) to obtain

$$
\partial_{t} e=-\nabla \cdot\left[\mathbf{q}_{K}+\mathbf{q}_{C}+\mathbf{q}_{D}\right]+\Gamma_{R}-\Gamma_{D} \equiv-\nabla \cdot \mathbf{q}+\Gamma .
$$

[1] Lattice Gas Methods for Partial Differential Equations, edited by G. D. Doolen (Addison-Wesley Publishing Company, Reading, MA, 1990).

[2] D. H. Rothman and S. Zaleski, Rev. Mod. Phys. 66, 1417 (1994).

[3] R. Benzi, S. Succi, and M. Vergassola, Phys. Rep. 222, 145 (1992).

[4] P. J. Hoogerbrugge and J. M. V. A. Koelman, Europhys. Lett. 19, 155 (1992).

[5] P. Español and P. Warren, Europhys. Lett. 30, 191 (1995); P. Español, Phys. Rev. E 53, 1572 (1996).

[6] J. M. V. A. Koelman and P. J. Hoogerbrugge, Europhys. Lett. 21, 363 (1993).

[7] E. S. Boek, P. V. Coveney, and H. N. W. Lekkerkerker, J. Phys., Condens. Matter 8, 9509 (1996); E. S. Boek, P. V. Coveney, H.N.W. Lekkerkerker, and P. van der Schoot, Phys. Rev. E 55, 3124 (1997).

[8] A. G. Schlijper, P. J. Hoogerbrugge, and C. W. Manke, J. Rheol. 39, 567 (1995).

[9] W. G. Madden, Y. Kong, C. M. Manke, and A. G. Schlijper, Int. J. Thermophys. 15, 1093 (1994).

[10] P. V. Coveney and K. E. Novik, Phys. Rev. E 54, 5134 (1996).

[11] J. van den Berg, D. ten Bosch, and M. H. Ernst, Master's thesis, University of Utrecht, 1996; and Research and Technical Services, Rijswijk, Holland, Internal Shell Report No.
SIEP-96-5071, 1996 (unpublished).

[12] P. Español, Phys. Rev. E 52, 1734 (1995).

[13] C. Marsh, Oxford University Internal Report, June 1996 (unpublished).

[14] C. A. Marsh and J. M. Yeomans, Europhys. Lett. 37, 511 (1997).

[15] L. E. Reichl, A Modern Course in Statistical Physics (University of Texas Press, Austin, 1980), Chap. 13.

[16] G. Grinstein, J. Appl. Phys. 69, 5441 (1991); D. H. Lee, G. Grinstein, and S. Sachdev, Phys. Rev. Lett. 64, 1927 (1990).

[17] T. R. Kirkpatrick, J. R. Dorfman, and J. V. Sengers, Annu. Rev. Phys. Chem. 45, 213 (1994).

[18] H. J. Bussemaker and M. H. Ernst, Phys. Rev. E 53, 5837 (1996).

[19] P. Resibois and M. de Leener, Classical Kinetic Theory of Liquids (Wiley, New York, 1977).

[20] S. Chapman and T. G. Cowling, The Mathematical Theory of Non-Uniform Gases, 3rd ed. (Cambridge University Press, Cambridge, England, 1970).

[21] J. T. Jenkins and M. W. Richman, Phys. Fluids 28, 3485 (1985).

[22] Please distinguish the bold face $\boldsymbol{\Gamma}$, denoting a point in phase space, from the light type $\Gamma$, denoting sources and sinks.

[23] S. R. de Groot and P. Mazur, Non-equilibrium Thermodynamics (North-Holland Publishing Company, Amsterdam, 1969). 
[24] E. G. D. Cohen, Fundamental Problems in Statistical Mechanics (North-Holland Publishing Company, Amsterdam, 1962), Vol. I, p. 110.

[25] N. G. Van Kampen, Stochastic Processes in Physics and Chemistry (North-Holland Publishing Company, Amsterdam, 1992), Chap. 8.

[26] U. Frisch et al., Ref. [1], p. 77.
[27] Statistical hydrodynamics of lattice gas automata, P. Grosfils, J. P. Boon, R. Brito, and M. H. Ernst, Phys. Rev. E 48, 2655 (1993).

[28] E. G. D. Cohen, I. M. de Schepper, and M. J. Zuilhof, Physica B \& C 127, 282 (1984); P. Verkerk, J. Westerweel, U. Bafile, L. A. de Graaf, W. Monfrooij, and I. M. de Schepper, Phys. Rev. A 40, 2860 (1989). 